UMA ABORDAGEM NÃO "STANDARD"

DA CONJECTURA DE EDGEWORTH

\title{
Carlos Antonio Bueno Raymundo
}

\author{
DISSERTAÇÃO APRESENTADA \\ $\mathrm{AO}$ \\ INSTITUTO DE MATEMÁTICA E ESTATÍSTICA \\ DA \\ UNIVERSIDADE DE SÃO PAULO \\ PARA \\ OBTENÇÃO DO GRAU \\ DE \\ MESTRE EM MATEMÁTICA \\ Área de Concentração: Análise Matemática \\ Orientador: Prof. Dr. João Carlos Prandini
}




\section{UMA ABORDAGEM NÃO "STANDARD" \\ DA CONJECTURA DE EDGEWORTH}

Carlos Antonio Bueno Raymundo

Este exemplar corresponde à redação final da dissertação apresentada por Carlos Antonio Bueno Raymundo, devidamente corrigida e aprovada pela Comissão Julgadora

São Paulo, 15 de maio de 1996

\section{Comissão Julgadora}

- Prof. Dr. João Carlos Prandini - IME-USP

- Prof. Dr. Ricardo Bianconi - IME-USP

- Prof. Dr. Paulo Picchetti - FEA-USP 


\section{RESUMO}

O objetivo deste trabalho é dar um tratamento matemático, via análise não "standard", para a conjectura de Edgeworth. Esta conjectura relaciona dois tipos de equilíbrio econômico, o "core" e o equilíbrio Walrasiano, com a seguinte afirmação: o crescimento do número de agentes em uma economia de trocas faz com que o "core" descresça, aproximando-se do equilíbrio competitivo ou Walrasiano.

\section{ABSTRACT}

The purpose of this work is to give a mathematical treatment on Edgeworth's conjecture using nonstandard analysis. This conjecture relates two types of economical equilibrium, the core and the Walrasian equilibrium. It says that as the number of traders in an exchange economy increases, the core shrinks to the set of competitive equilibria or Walras equilibria. 


\section{AGRADECIMENTOS}

Primeiramente gostaria de agradecer ao orientador Prof. Dr. João Carlos Prandini por todo o seu empenho para o término deste trabalho.

Gostaria de agradecer a Profa. Dra. Elza Furtado Gomide por toda ajuda que me prestou nestes anos todos.

Gostaria também de agradecer ao Prof. Dr. Ricardo Bianconi, a Profa. Dra. Zara Issa Abud, Prof. Dr. Jacob Zimbarg Sobrinho, Prof. Dr. Luciano Barbanti, e ao amigo Marcelo Rabbat pelo auxílio na revisão deste texto, ficando claro que os erros que porventura sejam encontrados devem-se exclusivamente a mim.

Gostaria de dedicar este trabalho a minha mulher Fatima, e agradecer-lhe por sua compreensão e carinho ao longo destes anos.

E agradeço a Deus por ter conseguido chegar ao final deste trabalho. 


\section{Conteúdo}

1 Economias de pura troca 3

1.1 Existência do Equilíbrio Walrasiano . . . . . . . . . . . . . . . 3

1.2 O "Core" e o Equilíbrio Walrasiano . . . . . . . . . . . . . . . 12

1.3 Réplicas de uma economia e teorema limite. . . . . . . . . . . . . 20

2 Economias de trocas não "standard" 22

2.1 A equivalência entre o "core" e o equilíbrio Walrasiano . . . . . . . . . . 23

2.2 Réplicas de uma economia . . . . . . . . . . . . . . . . . . . 37

A "Commodities" e relações de preferências 44

B S-Convexidade: definições e lemas $\quad 52$

C Elementos de análise não "standard" $\quad 57$

$\begin{array}{ll}\text { Bibliografia } & 65\end{array}$ 


\section{Introdução}

O objetivo deste trabalho é a fundamentação matemática da conjectura de Edgeworth, que relaciona dois tipos de equilíbrios econômicos que chamaremos de equilíbrio Walrasiano (ou equilíbrio competitivo), e "core" (ou núcleo econômico). O equilíbrio competitivo se dá quando obtemos um vetor de preços e uma redistribuição dos bens da economia que satisfaz a totalidade dos agentes eccnômicos; o segundo diz respeito ao conjunto das redistribuições de bens que não podem ser melhoradas por qualquer outra redistriuição de bens promovida por subconjuntos de agentes econômicos objetivando maximizar suas preferências. A conjectura de Edgeworth relaciona estes dois tipos de equilíbrio com a seguinte afirmação:

"O crescimento do número de agentes numa economia realizando trocas de bens, buscando maximizar suas preferências, faz com que o "core" decresça aproximando-se do equilíbrio Walrasiano."

Neste trabalho serão apresentadas quatro maneiras diferentes de se abordar a conjectura de Edgeworth:

a) O teorema clássico de Aumann,

b) O teorema clássico de Debreu-Scarf (teorema limite),

c) A abordagem de Brown and Robinson para a equivalência do "core" e o equilíbrio Walrasiano via análise não "standard", 
d) A abordagem de Brown anda Robinson para réplicas economicas relacionando o "core" e o equilíbrio Walrasiano via análise não "standard".

No capítulo 1 são apresentados modelos matemáticos para economias de pura troca, os resultados clássicos que provam a existência do equilíbrio Walrasiano e a conjectura de Edgeworth.No capítulo 2 abordaremos a conjectura de Edgeworth e daremos a sua prova utilizando como ferramenta matemática a análise não "standard". 


\section{Capítulo 1}

\section{Economias de pura troca}

Este capítulo esta dividido em três seções. A primeira trata da existência do equilíbrio competitivo, a segunda da relação entre o equilíbrio competitivo e o "core", e a terceira relaciona o "core" e o equilíbrio walrasiano através de réplicas econômicas. Iniciaremos as seções definindo um modelo para economia de trocas. Todas as definições e resultados apresentados nas três seções serão, na medida do possível, acompanhados de exemplos. Antes de iniciarmos a seção 1 introduziremos alguma notações:

$$
\begin{aligned}
R_{+}^{n} & =\left\{x=\left(x_{1}, \ldots, x_{n}\right) \in R^{n}: x_{i} \geq 0, i=1, \ldots, n\right\} \\
S_{+}^{n-1} & =\left\{x=\left(x_{1}, \ldots, x_{n}\right) \in R_{+}^{n}: \sum_{i=1}^{n} x_{i}=1\right\}
\end{aligned}
$$

Dado um conjunto $A \subset R_{+}^{n}, \mathcal{P}(A)$ indica o conjunto das partes de $A$. Dados $x, y \in R^{n}$, escreveremos:

a) $x \geq y$ para indicarmos que $x_{i} \geq y_{i}$, para todo $i=1, \ldots, n$,

b) $x \gg 0$ para indicarmos que $x_{i}>0$, para todo $i=1, \ldots, n$.

\subsection{Existência do Equilíbrio Walrasiano}

\section{Economias de Pura Troca}

Definição 1.1 Uma economia de pura troca ou simplesmente uma economia de trocas é um conjunto finito $\left.\varepsilon=\left\{e_{i}, \succeq_{i}\right) ; i=1, \ldots, k\right\}$, onde $e_{i} \in R_{+}^{n} e \succeq_{i}$ é uma relação binária 
sobre $R_{+}^{n}$. O conjunto $R_{+}^{n}$ será denominado espaço das "commodities".Assim, economia de trocas consiste na existência de $k$ indivíduos ou agentes, denominados "traders" ou. consumidores, possuidores de uma cesta de bens e uma preferência e que procuram realizar trocas desses bens com o intuito de obterem a maior satisfação possível de consumo ou riqueza.

O elemento $e_{i}=\left(x_{1}^{i}, \ldots, x_{n}^{i}\right) \in R_{+}^{n}$ representa a cesta de bens iniciais do consumidor $i$, onde $x_{j}^{i} \geq 0$ e $x_{j}^{i}$ representando a quantidade do $j$-ésimo bem, que chamaremos de "commodity"; isto por que estão pressupostos além da quantidade, data e local da disponibilidade do bem.

Desta forma, a quantidade dos bens disponíveis na economia é dada pela $\sum_{i=1}^{k} e_{i}$.

A preferência $\succeq_{i}$ do consumidor $i$, é uma relação binária sobre $R_{+}^{n}$, tal que se $(x, y) \in \succeq_{i}$, diremos que o consumidor $i$ prefere a cesta $x$ à cesta $y$ e escreveremos $x \succ_{i} y$, quando o consumidor $i$ for indiferente entre $x$ e $y$ escreveremos $x \sim_{i} y$. Reunimos as duas relações para o agente $i$ no símbolo $\succeq_{i}$.

Dizer que as preferências são contínuas, significa que $\succeq_{i}$ é um subconjunto fechado $\mathrm{em} R_{+}^{n} \times R_{+}^{n}$.

No decorrer do capítulo, exigiremos certas propriedades sobre preferências, estas serão apresentadas de forma sucinta objetivando os resultados que seguem; maiores detalhes sobre tais propriedades poderão ser encontradas no apêndice A.

Sistema de preços e o "budget set"

Sistema de Preços

Definição 1.2 Um vetor de preços, $p$, é um elemento de $R_{+}^{n}$, onde $p=\left(p_{1}, \ldots, p_{n}\right)$ é tal que $p_{j}, j=1, \ldots, n$, é preço de uma unidade do bem $j, j=1, \ldots, n$. Assim, dada uma cesta de "commodities" $x \in R_{+}^{n}$, o preço da cesta $x$ é dado por p.x $=\sum_{i=1}^{n} p_{i} x_{i}$. 


\section{"Budget Set"}

Definição 1.3 O "Budget Set" do consumidor $i$, segundo o sistema de preços p, é o conjunto

$$
\beta\left(p, e_{i}\right)=\left\{x \in R_{+}^{n}: p . x \leq p \cdot e_{i}\right\} .
$$

Isto é, dado um sistema de preços, $p$, o consumidor i pode consumir toda cesta de "commodities" cujo preço é menor ou igual ao preço dos bens que possui.Chamaremos de riqueza do "trader" $i$, segundo o vetor de preços $p, i=l, \ldots, k$, ao número p.e ${ }_{i}$.

\section{Correspondência de Demanda}

Definição 1.4 Para cada consumidor $i$, com dotaçôes iniciais $e_{i}$, chamamos de correspondência de demanda do consumidor $i$, a aplicação

$$
\begin{aligned}
\varphi_{i}: R_{+}^{n} \times R_{+}^{n} & \rightarrow \mathcal{P}\left(R_{+}^{n}\right) \\
\left(p, e_{i}\right) & \mapsto\left\{x \in \beta\left(p, e_{i}\right): \forall y \in \beta\left(p, e_{i}\right) x \succeq y\right\}
\end{aligned}
$$

Assim, a correspondência de demanda é uma correspondência (função multívoca, isto é, associa pontos a conjuntos) que associa, para cada consumidor $i$, com dotações inciais $e_{i}$ os elementos maximais em $\beta\left(p, e_{i}\right)$ segundo a preferência $\succeq_{i}$.

A correspondência de demanda, $\varphi$, goza das seguintes propriedades:

a) $\varphi$ é homogênea de grau zero, isto é:

$$
\forall \lambda>0, \varphi\left(\lambda p, \lambda e_{i}\right)=\varphi\left(p, e_{i}\right), \text { pois } \beta\left(\lambda p, \lambda e_{i}\right)=\beta\left(p, e_{i}\right)
$$

Portanto podemos tomar $p \in S_{+}^{n-1}=\left\{p \in R_{+}^{n}: \sum_{i=1}^{n} p_{i}=1\right\}$.

Doravante admitiremos que $\varphi: S_{+}^{n-1} \times R_{+}^{n} \rightarrow \mathcal{P}\left(R_{+}^{n}\right)$. 
b) Se $\succeq$ é localmente não saciada, (isto é, dado $x \in R_{+}^{n}$ e $\varepsilon \in R, \varepsilon>0, \exists y \in R_{+}^{n}$ com $\|x-y\|<\varepsilon$ e $y \succeq x\})$, então $p . \varphi\left(p, e_{i}\right)=p . e_{i}$, pois, $\forall x \in \beta\left(p, e_{i}\right)$, tal que $p . x<p . e_{1}$, existe $x^{\prime} \succeq x$ com $p . x^{\prime}<p . e_{i}$.

A propriedade b) nos diz que com preferências localmente não saciadas, os "agentes" consomem toda sua riqueza na busca de bens que maximizem suas preferências.

c) Se $\succeq$ é estritamente convexa, então $\left|\varphi\left(p, e_{i}\right)\right| \leq 1$, onde $\left|\varphi\left(p, e_{i}\right)\right|$ denota o número de elementos de $\varphi\left(p, e_{i}\right)$.

Observação: Dizemos que $\succeq$ é estritamente convexa quando dadas cestas $x, y$ com $x \sim_{i} y$ (le-se: o "agent.e" $i$ é indiferent.c a consumir a cesta $x$ ou $y$ ), $x \neq y, 0<\lambda<1 \Rightarrow$ $\lambda x+(1-\lambda) y \succ x)$.

Para provar a afirmação () acima, basta observar que se $x, y \in \varphi\left(p, e_{i}\right)$ e $x \neq y, x \sim_{i} y$, então para todo $\lambda$, com $0<\lambda<1$ tem-se $\lambda x+(1-\lambda) y \succ x$ e como $p \cdot(\lambda x+(1-\lambda) y)) \leq$ $p . e_{i} \Rightarrow \lambda x+(1-\lambda) y \in \varphi\left(p, e_{i}\right)$; o que nos leva a uma contradição, pois $x$ é maximal em $\varphi\left(p, e_{i}\right)$ para $\succeq$.

\section{EXEMPLO 1}

Seja $\varepsilon$ uma economia de trocas com dois bens e dois agentes com preferências localmente não saciadas (vide apêndice A). Os bens do agente, $i, i=1,2$, será denotado por $e_{i}=\left(e_{i 1}, e_{i 2}\right)$, onde $e_{i j}$ representa a quantidade do bem $j$, do agente $i$.

Geometricamente, $\varepsilon$ pode ser representada pela figura abaixo

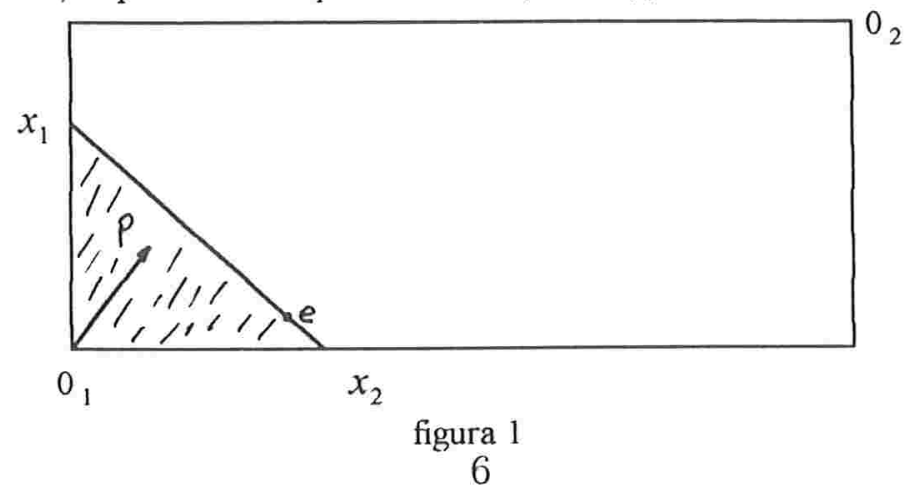


O eixo horizontal representa o bem 1 e o eixo vertical, o bem 2 . O comprimento da caixa é dado por $e_{11}+e_{21}$ e sua altura é dada por $e_{12}+e_{22}$, em que $e_{11}$ e $e_{21}$ representam a quantidade do bem 1 do consumidor 1 e 2 , respectivamente, e $e_{12}$ e $e_{22}$ a quantidade do bem 2 do consumidor 1 e 2 , respectivamente.

As quantidades do bem 1 e do bem 2 para o consumidor 1 , são dadas considerando-se como origem o ponto $0_{1}$.

As quantidades do bem 1 e do bem 2 para o consumidor 2 , são dadas considerando-se como origem o ponto $0_{2}$.

O ponto $e$, no interior da caixa representa as cestas $e_{1}, e_{2}$, lidos considerando-se as origens $0_{1}, 0_{2}$, respectivamente.

Dado um vetor de preços $p=\left(p_{1}, p_{2}\right)$, o "budget set" do consumidor 1 , por exemplo, é dado pela região hachurada.

A imagem da correspondência de demanda do consumidor 1, com preferência localmente não saciada, $\varphi\left(p, e_{1}\right)$, está contida no segmento com extremos $x_{1}, x_{2}$, que representam respectivamente a quantidade máxima do bem 1 e do bem 2 , caso o consumidor 1 opte por consumir apenas um dos bens disponíveis segundo o vetor de preços em vigor.O seguimento com estremos $x_{1}, x_{2}$ está contido no hiperplano $H_{p}=\left\{x \in R_{+}^{2}: p x=p e\right\}$.

Chamaremos a representação acima para a economia $\varepsilon$ de caixa de Edgeworth.

\section{Função utilidade}

Definição 1.5 Uma função utilidade é uma aplicaçâo, u, que a cada elemento $x \in R_{+}^{n}$ associa um nível de satisfação u. $(x)$.

Isto é, $u: R_{+}^{n} \rightarrow R$ e cada curva de nível da função $u$, caracteriza um nivel de satisfação do consumidor, e para cada cesta $x, y$ pertencentes a uma mesma curva de nível, diremos que o consumidor é indiferente a consumir a cesta $x$ ou $y$, ou seja, ambas dão ao consumidor a mesma satisfação. 


\section{EXEMPLO 2}

Consideremos novamente a caixa de Edgeworth do exemplo 1, mas suponha agora que as preferências dos agentes sejam convexas (vide apêndice A).

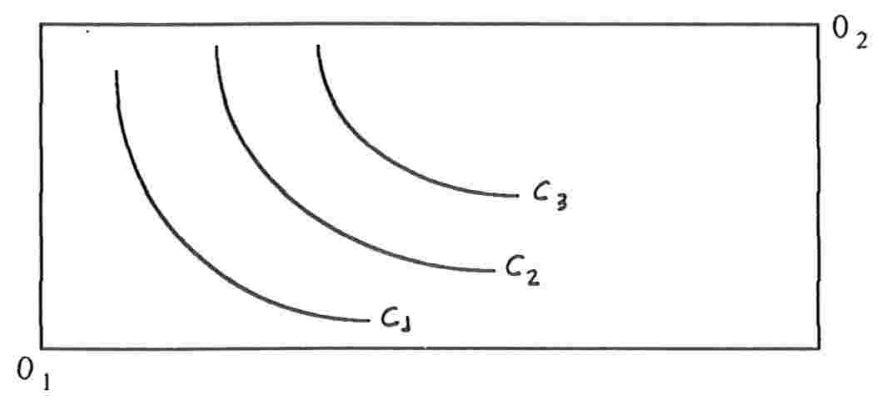

figura 2

Seja $u_{1}$ uma função utilidade para o consumidor 1 , a partir da origem $0_{1}$. Suponha que as curvas $c_{i}, i=1,2,3$, da representem algumas das curvas de nível da função utilidade $u_{1}$. Isto é, para cada $x \in c_{i}, u_{1}(x)=c_{i}$.

Chamaremos as curvas de nivel de uma função utilidade de curvas de indiferença.

Definição 1.6 Diremos que uma preferência $\succeq_{i}$ e uma utilidade $u_{i}$ do consumidor $i$ estão associadas ou que $\succeq_{i}$ representa $u_{i}$ (e escrevemos $\succeq_{u_{i}}$ ) ou que $u_{i}$ representa $\succeq_{i}$ (e escrevemos $u_{\succeq_{i}}$ ) caso para todo $x, y \in R_{+}^{n}, x \succeq_{i} y \Leftrightarrow u_{i}(x) \geq u_{i}(y)$.

Teorema 1.1 Sejam $X$ um conjunto contável de segunda ordem, $\succeq_{i}$ relaçâo binária sobre $X$ representando a preferência do consumidor $i$. suponha $\succeq_{i}$ continua, então existe uma função utilidade contínua, $u_{i}$, representando $\succeq_{i}$.

Prova: Vide Continuity properties of paretian utility, Debreu,G.,I.E.R., 5 sept. 1964.

Observação: Um conjunto $X$ é dito contável de segunda ordem se existirem abertos $A_{n}$, $n \in N$, tais que para todo $A \subset X$ existe $I \subseteq N$, com $A=\bigcup_{i \in I} A_{i}$. Todo subconjunto fechado de $R^{n}$ é contável de segunda ordem quando considerado com a topologia induzida pela topologia usual. 
Definição 1.7 Chamamos de pontos factiveis ou trocas factiveis para $\varepsilon$ ao conjunto

$$
F A=\left\{x \in R_{+}^{n k}, \sum_{i=1}^{k} x^{i} \leq \sum_{i=1}^{k} e_{i}, x^{i}, e_{i} \in R_{+}^{n}\right\} .
$$

OFA é o conjunto das cestas cujas quantidades nào ultrapassam o total de bens disponíveis na economia.

Definição $1.8 O \operatorname{par}(x, p) \in R_{+}^{n k} \times S_{+}^{n-1}$ é chamado equilíbrio walrasiano (ou equilíbrio competitivo) para a economia $\varepsilon$ se:

a) $x_{i} \in \varphi\left(p, e_{i}\right), i=1,2, \ldots, k$;

b) $\sum_{i=1}^{k} x_{i}=\sum_{i=1}^{k} e_{i}$, isto é, a cesta $x_{i}$ maximiza a preferência $\succeq_{i}$ do consumidor $i=1,2, \ldots, k$, e a demanda é igual à oferta dos bens.

Teorema 1.2 (existência do equilíbrio walrasiano) Seja $\varepsilon$ uma economia de trocas com $k$ consumidores, tal que suas preferências sejam contínuas, estritamente convexas e estritamente crescentes (ou monótonas); suponha também que $e_{i} \gg 0$, para $i=1, \ldots, k$. Então existe o equilíbrio walrasiano.

Prova: Vide Introdução à Economia Matemática, Aloísio P. Araújo, p.73 (14o Colóquio Brasileiro de Matemática).

A demonstração do teorema acima pode ser feita utilizando-se o teorema do ponto fixo de Brouwer ou mais geralmente o teorema do ponto fixo de Kakutani.

\section{EXEMPLO 3}

Considere a caixa de Edgeworth do exemplo 1. Suponha agora que as preferências dos consumidores são contínuas e convexas (vide apêndice A).

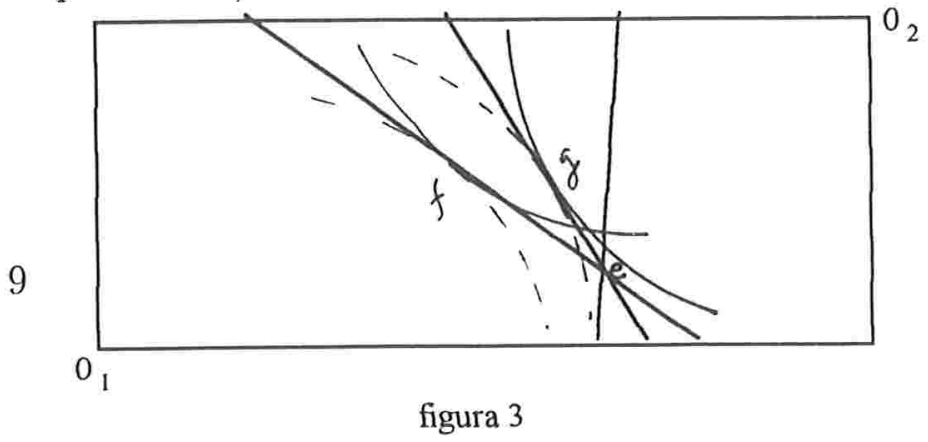


Suponha que as curvas tracejadas representem as curvas de indiferença do consumidor 2, e as de traçado contínuo a as curvas de indiferença do consumidor 1 .

Sejam $p_{1}, p_{2}, p_{3}$ três vetores de preços distintos para a economia descrita acima, cada vetor de preços determina um hiperplano $H_{p_{i}}=\left\{x \in R_{+}^{2}: p x=p e\right\}, i=1,2,3$; tal que considerando-se a origem $0_{1}$, os pontos que estão à esquerda de $H_{p_{i}}$ pertencem ao Budget set do consumidor 1 e os pontos que estão à direita de $H_{p}$, pertencem ao budget set do consumidor 2 .

A imagem da função de demanda do consumidor 1 e 2 considerando-se suas respectivas origens, está contida no hiperplano $H_{p_{i}}, i=1,2,3$.

O equilíbrio walrasiano se dá em um ponto $x \in H_{p_{i}}$, para algum $i=1,2,3$, que seja ponto de tangência mútua de alguma curva de indiferença do consumidor 1 e 2 respectivamente. Os pontos $f, g$ são equilíbrios warlrasiano para a economia de trocas representada pela caixa.

O hiperplano $H_{p_{3}}$ não possui nenhum ponto que seja ponto de tangência mútua às curvas de indiferença dos consumidores 1 e 2 portanto com o vetor de preços $p_{3}$ não temos um equilíbrio walrasiano para a economia acima descrita.

De um modo geral, um ponto $m$ pertencente a caixa será um ponto de equilíbrio se a reta que liga as dotações iniciais, $e$, dos agentes 1 e 2 respectivamente, ao ponto $m$ é tangente às curvas de indiferença de ambos os consumidores, que passem por $m$.

\section{EXEMPLO 4}

Seja $\left.\varepsilon=\left\{x, \succeq_{i}\right), i=1,2\right\}$, onde $x \in R_{+}^{2}, \succeq_{i}, i=1,2$, relação binária em $R_{+}^{2}$. Suponha que a relação de preferência $\succeq_{i}, i=1,2$, seja representada pela função utilidade $u_{i}: R_{+}^{2} \rightarrow R$, dada por $u_{i}\left(x_{1}, x_{2}\right)=x_{1}^{\alpha_{i}} x_{2}^{1-\alpha_{i}}$, onde $0<\alpha_{i}<1$ (a função utilidade $u_{i}$, $i=1,2$, assim definida é chamada de função utilidade de Cobb-Douglas). 
A relação de preferência representada por $u_{i}$ é fortemente convexa (vide apêndice A), sendo assim a correspondência de demanda do consumidor, $i$, determina a cada vetor de preços $p$ uma função, que chamaremos de função de demanda individual. Seja $p=\left(p_{1}, p_{2}\right)$ um sistema de preços, com $p_{i}>0, i=1,2$. Temos então que função de demanda individual, $\varphi_{i}$, do consumidor $i$ será dada por

$$
\varphi(p, e(i))=\left(\frac{\alpha_{i} p \cdot e(i)}{p_{1}}, \frac{\left(1-\alpha_{i}\right) p \cdot e(i)}{p_{2}}\right), \quad e(i)=\left(e_{1}(i), e_{2}(i)\right) .
$$

Observação: Obtemos a função $\varphi$ da seguinte maneira: Dado um sistema de preços $p$, sendo $\succeq_{i}$ fortemente convexa (vide apêndice A), podemos encontrar o maximo da função utilidade $u_{i}$, que representa a preferência $\succeq_{i}$, restrita à superfície $M=\left\{x \in R_{+}^{2}: p_{x}=\right.$ $\left.p e_{i}\right\}=g^{-1}\left(p e_{i}\right)$, em que $g: R_{++}^{2} \rightarrow R$, que a cada $x$ associa $g(x)=p x$.

Faremos isto aplicando o teorema dos multiplicadores de Lagrange a $u_{i}$ restrita a $M$. Temos então:

$$
u_{i}\left(x_{1}, x_{2}\right)=x_{1}^{\alpha_{i}} x_{2}^{\left(1-\alpha_{i}\right)} .
$$

Afim de facilitar os cálculos, seja $v_{i}\left(x_{1}, x_{2}\right)=\ln u_{i}\left(x_{1}, x_{2}\right)=\alpha_{i} \ln x_{1}+\left(1-\alpha_{i}\right) \ln x_{2}$. Temos que o gradiente de $v_{i}\left(x_{1}, x_{2}\right)$ é dado por $\nabla v_{i}\left(x_{1}, x_{2}\right)=\left(\frac{\alpha_{i}}{x_{1}}, \frac{\left(1-\alpha_{i}\right)}{x_{2}}\right)$, e o gradiente de $g$ é dado por $\nabla g\left(x_{1}, x_{2}\right)=\left(p_{1}, p_{2}\right)$; e pelo teorema dos multiplicadores de Lagrange temos que um ponto $x \in M$ é ponto crítico de $v_{i}$ restrita $M$, e portanto um ponto crítico de $u_{i}$ restrita a $M$, se existir $\lambda$ real tal que $\nabla v_{i}\left(x_{1}, x_{2}\right)=\lambda \nabla g\left(x_{1}, x_{2}\right)$, isto é, se

1) $\frac{\alpha_{i}}{x_{i}}=\lambda p_{i} \mathrm{e}$

2) $\frac{\left(1-\alpha_{i}\right)}{x_{2}}=\lambda p_{2}$, tal que

3) $p x=p e_{i}$,

vem daí que $\alpha_{i}=\lambda p_{1} x_{1}$ e, de 2) e 3) temos $\left(1-\alpha_{i}\right)=\lambda p_{2} x_{2}=\lambda p e_{i}-\lambda p_{1} x_{1}$ e, portanto, $\lambda=\frac{1}{p e_{i}}$. 
Temos então que substituindo $\lambda$ e 1) e 2) obtemos

$$
x=\frac{\alpha_{i} p e_{i}}{p_{1}} \quad \text { e } \quad y=\frac{\left(1-\alpha_{i}\right) p e_{i}}{p_{2}} .
$$

Chamaremos de função de demanda total a função:

$$
\phi: S_{+}^{n} \rightarrow R \text { dada por } \phi=\sum \varphi(p, e(i))
$$

Seja $E(p)=\phi-\sum e(i)$ função excesso de demanda. Temos que a solução do sistema

$$
\begin{aligned}
& {\left[\begin{array}{cc}
b_{11}-e_{1}(1)+e_{1}(2) & b_{12} \\
b_{21} & b_{22}-e_{2}\left((1)+e_{2}(2)\right)
\end{array}\right]\left[\begin{array}{l}
p_{1} \\
p_{2}
\end{array}\right]=\left[\begin{array}{l}
0 \\
0
\end{array}\right] \text {, em que }} \\
& b_{k j}=\alpha_{i} e_{j}(1)+\left(1-\alpha_{i}\right) e_{j}(2)
\end{aligned}
$$

é um vetor $p *$ não nulo que iguale a oferta total de bens a demanda tot.al de bens.

\subsection{O "Core" e o Equilíbrio Walrasiano}

Nesta seção definiremos uma forma mais fraca de equilíbrio para economias de trocas, que chamaremos de "core", e em seguida enunciaremos resultados que relacionam este tipo de equilíbrio ao equilíbrio "Walrasiano". Por fim, apresentaremos um exemplo de economia de trocas onde, de forma geométrica, poderemos visualizar a relação existente entre os dois tipos de equilíbrio.

A definição de "core" de uma economia, num contexto mais geral, é dado como segue: O "core" de uma economia consiste do todos os estados da economia que não podem ser melhorados por qualquer coalizão de agentes econômicos. Um estado para uma economia de trocas é uma redistribuição dos bens na economia de tal forma que a soma de todos os bens redistribuidos seja igual a soma dos bens disponíveis, isto é, não há criação de novos bens e tão pouco destruição dos bens já existentes. 
Os principais resultados relacionando o "core" ao equilíbrio "Walrasiano" serão dados por dois teoremas, cada um deles procura dar, à sua maneira, sentido à conjectura de Edgeworth, cujas demonstrações serão omitidas; indicaremos porém, onde tais demonstrações podem ser encontradas.

Para o que segue, vamos refrasear a definição de economia de trocas de forma mais conveniente.

Definição 1.9 Uma economia de trocas é uma aplicação $\varepsilon$ definida em um conjunto finito A. que chamaremos conjunto dos agentes economicos, tomando valores em $P \times R_{+}^{l}$, que chamaremos conjunto das características dos agentes.

O conjunto $R_{+}^{l}$ denota o espaço das "commodities".

$O$ conjunto $P$ denota o conjunto das relaçôes de preferências sobre $R_{+}^{l}$.

Assim para cada $a \in A$, temos $\varepsilon(a)=\left(\succ_{a}, e_{a}\right)$, onde $\succ_{a}$ é a relaçâo de preferência do agente a sobre $R_{+}^{l}$ e e e a l-upla dos bens iniciais ou dotações iniciais do agente a. Desta forma os bens disponiveis na economia $\varepsilon$ é dado pelo $\sum_{a \in A} e_{a}$.

Definição 1.10 Uma troca para a economia $\varepsilon$ é uma aplicação $f: A \rightarrow R_{+}^{l}$ que associa a cada agente a uma nova cesta de bens $f(a) \in R_{+}^{l}$, tal que $\sum_{a \in A} f_{a} \leq \sum_{a \in A} e_{a}$, isto é, nào há criação de novos bens na economia. Chamaremos de alocação ou um estado para. $\varepsilon$ a toda função de troca tal que $\sum_{a \in A} f_{a}=\sum_{a \in A} e_{a}$.

Definição 1.11 Chama-se coalizão a qualquer subconjunto de A. Dadas as alocaçôes $f$ e $g$, diremos que a alocação $g$ domina a alocação $f$ via coalizão $S$ se:

i) $g_{a} \succ_{a} f_{a}$, para todo $a \in S$;

ii) $\sum_{a \in S} g_{a}=\sum_{a \in S} e_{a}$.

Dada uma alocação $f$, se existir $g$ satisfazendo i) e ii) diremos que a alocação $f$ foi melhorada via coalizão $S$. O conjunto das alocaçàes ou estados para $\varepsilon$ que não podem ser melhoradas por qualquer coalizão será chamado de "core" e denotado por $\ell(\varepsilon)$. 
Abaixo, repetimos a definição de equilíbrio Walrasiano.

Definição 1.12 Uma troca $f$ para uma economia é chamada de alocaçào Walrasiana (ou equilibrio competitivo) se:

a) $f_{a}$ é maximal para $\succ_{a}$ em $\beta_{a}(p)=\left\{x \in R_{+}^{p}: p . x \leq p . e_{a}\right\}$, para todo $a \in A$;

B) $\sum_{a \in A} f_{a}=\sum_{a \in A} e_{a}$.

$O$ conjunto de todas as alocações Walrasianas será denotado por $\mathcal{W}(\varepsilon)$.

Proposição $1.1 \mathcal{W}(\varepsilon) \subset \ell(\varepsilon)$ para toda economia $\varepsilon$.

Prova: Vide Handbook of Matematical Economics, vol 2. pag. 836.

Teorema 1.3 O "core" de toda economia de trocas cujas preferências dos agentes são: contínuas, convexas e monotônicas; é não vazio.

Prova: Imediata, pois se $\ell(\varepsilon)=\emptyset \Rightarrow \mathcal{W}(\varepsilon)=\emptyset$, o que contradiz o teorema 1.2 , seção 1 .

Ótimo de Pareto

Definição 1.13 Diremos que uma alocação $f$ é um ótimo de Pareto se:

a) $f \in F A$;

b) Não existe nenhuma alocaçâo $f^{\prime}$ tal que $f^{\prime}(a) \succeq_{a} f(a)$, para todo $f^{\prime}(a) \succ_{a} f(a)$ para pelo menos um a.

Podemos interpretar a definição acima do seguinte modo: uma alocação $f$ não é um ótimo de Pareto se for possivel melhorar a situação de pelo menos um agente econômico sem prejuízo aos demais.

A seguir daremos alguns exemplos que ajudarão no esclarecimento da definição de ótimo de Pareto. 


\section{EXEMPLO 5}

Considere a economia de trocas dada no exemplo l, seção 1 .

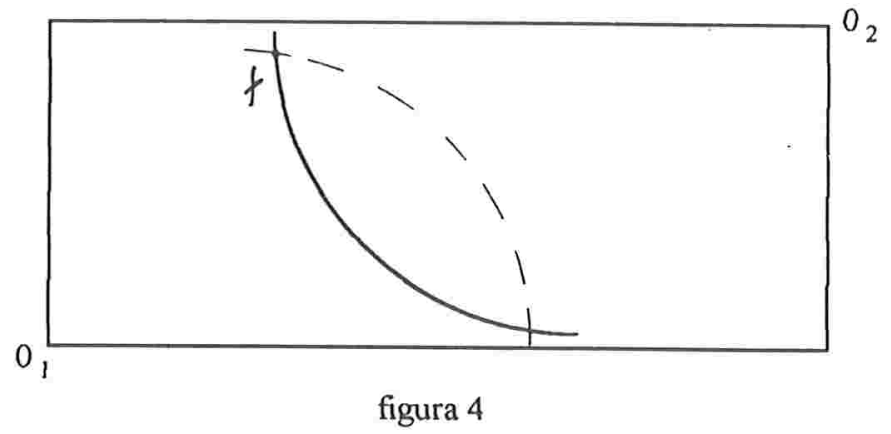

Observando que o agente 1 melhora sua situação se consumir cestas que estão acima da curva $c_{1}$ e o agente 2 melhora sua situação se consumir cestas que estão abaixo da curva $c_{2}$, temos que a alocação $f$ dada acima não é um ótimo de Pareto pois se caminharmos sobre curva $c_{1}$ no sentido anti-horário a situação do agente 1 permanece inalterada, (pois toda cesta sobre $c_{1}$ lhe proporciona a mesma satisfação de consumo); já a situação do agente 2 foi melhorada, pois tais cestas estão abaixo da cesta $c_{2}$ e portanto the proporcionam maior satisfação de consumo; de forma análoga, se caminharmos no sentido horário sobre a curva $c_{2}$, a situação do agente 2 permanece inalterada e a situação do agente 1 será melhorada.

\section{EXEMPLO 6}

Considere a economia de trocas dada no exemplo 1 , seção 1 .

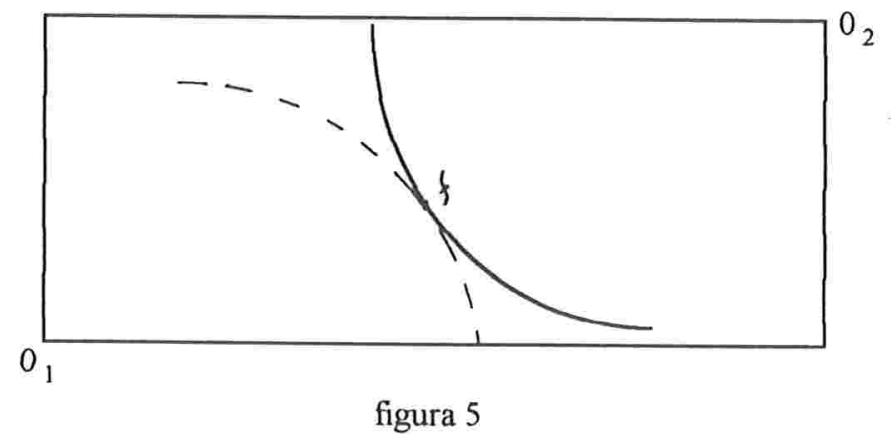

A alocão $f$ neste exemplo é um ótimo de Pareto, pois se caminharmos sobre a curva 
$c_{1}$ no sentido anti-horário o consumidor 2 terá sua situação piorada, pois este passará a consumir cestas que estão acima da curva $c_{2}$; de forma análoga, se caminharmos sobre a curva $c_{2}$ no sentido horário a situação do agente 1 será piorada. Temos então que para a economia de trocas representada pela caixa de Edgeworth acima, os ótimos de Pareto são os pontos de tangência mútua das curvas de indiferença dos agentes 1 e 2, respectivamente.

\section{EXEMPLO 7}

Considere, novamente, a economia de trocas dada no exemplo 1. seção 1.

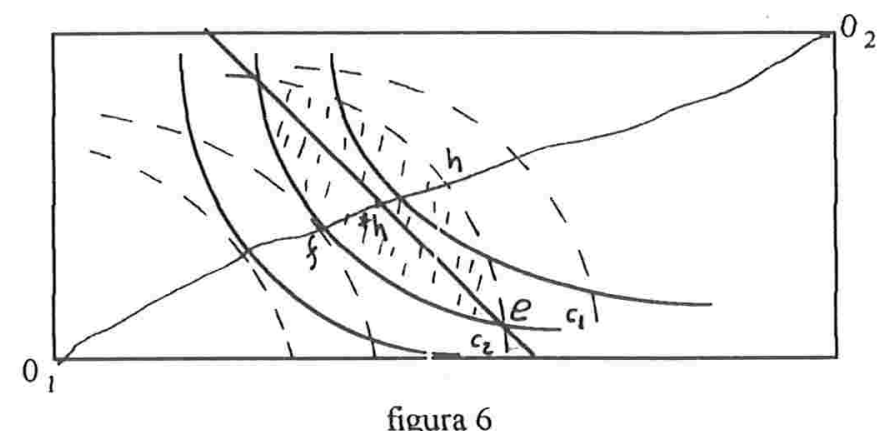

figura 6

Como vimos no exemplo 6, alocações dadas pelos pontos de tangencia mútua das curvas de indiferença dos agentes 1 e 2, são os ótimos de Pareto. Lembramos que o ponto e na caixa representa as dotações iniciais dos agentes 1 e 2 quando lido considerando-se as origens $\mathrm{O}_{1}$ e $0_{2}$, respectivamente. Lembramos também que o consumidor 1 melhora suas dotações iniciais se consumir as cestas que estão acima da curva $c_{1}$ e o consumidor 2 melhora suas dotações iniciais se consumir as cestas que estão abaixo da curva $c_{2}$; desta forma as alocações que melhoram as utilidades dos consumidores 1 e 2 , simultaneamente, serão aquelas que estão na região hachurada, pois fora desta região haverá perda de utilidade para um dos dois agentes. Dado que a economia acima possui apenas dois consumidores, temos que são possiveis apenas dois modos de se formar uma coalizão, a coalizão de um agente ou a coalizão de dois agentes.

Consideremos, primeiramente, a coalizão de dois agentes. Como visto acima, as alocações que melhoram simultaneamente a situação dos dois agentes serão aquelas que 
se encontram na região hachurada; estas por sua vez, se não forem alocações pertencentes ao "core" serão bloqueadas pelas alocações do "core".

Afirmamos que com preferências convexas toda alocação do "core" é um ótimo de Pareto.

Prova: Seja $Y$ uma alocação no "core" da economia tal que $Y$ não é um ótimo de Pareto. Sem perdas de generalidades, existe uma alocação $X$ tal que $X_{2} \succeq_{2} Y_{2}$ e $X_{1} \succ_{1} Y_{1}$, sendo as preferências dos agentes convexas, existe $\delta>0$ tal que para todo $Z \in B_{\delta}(X)$ temos $Z_{1} \succ_{1} Y_{1}$; desta forma, podemos obter uma alocação $Z^{\prime} \in B_{\delta}(X)$ tal que $Z_{1}^{\prime}$ possua decréscimos positivos de bens em relação a $X_{1}$ e $Z_{2}^{\prime}$ possua acréscimos positivos de bens transferidos do agente 1 na troca de $X_{1}$ por $Z_{1}^{\prime}$.

Desta forma, $Z_{2}^{\prime} \succ_{2} X_{2} \Rightarrow Z_{2}^{\prime} \succ_{2} Y_{2}$ e, portanto, $Z^{\prime}$ melhora a alocação $Y$, o que contradiz o fato de $Y$ estar no "core" da economia. Temos, então, que toda alocação no "core" da economia é um ótimo de Pareto.

Consideremos agora a coalizão de um agente. Vamos supor, sem perdas de generalidade, que a coalizão de um único agente será representada pelo agente 1. A alocação para o agente 1 será dada pelo ponto $e$ da caixa, pois esta alocação lhe garante o nível de satisfação representado pela totalidade de seus bens. Observe que o ponto $f$ melhora a situação do agente 2 sem provocar perda de utilidade para o agente 1 , e $f$ por sua vez não pode ser melhorada por nenhuma outra alocação pois esta é um ótimo de Pareto e sua melhoria implicaria uma coalizão de dois agentes e, como visto acima, nos levaria a uma contradição. Vem daí que $f$ é uma alocação no "core" da economia. De forma análoga, a alocação $h$ estará no "core" da economia, e neste caso, $h$ bloquearia a coalizão dada pelo agente 2. Desta forma, as alocações que não poderão ser melhoradas serão aquelas que pertencem à curva de extremos $f, h$, pois estas, como visto, melhoram a situação dos dois agentes simultaneamente e por serem alocações ótimos de Pareto não podem ser 
melhoradas por nenhuma outra alocação da região.

Para completar, seja $p$ um sistema de preços, seja $H_{p}$ o hiperplano gerado por $p$, como visto na seção 1, a imagem da função de demanda do consumidor 1 e 2 está contida em $H_{p}$ e portanto os agentes procurão consumir as cestas pertencentes a $H_{p}$, pois estas maximizam as preferências dos dois ager.tes segundo o vetor de preços $p$. Dentre as cestas pertencentes a $H_{p}$, a única que não pode ser melhora é a $h *$, pois é um ótimo de Pareto e está no "core" da economia e mais, tal cesta constitui um equilíbrio Walrasiano para a economia segundo o sistema de preços $p$.

Observação: A curva que percorre a diagonal da caixa é chamada de curva de expansão e contém todas as alocações que são um. ótimo de Pareto; observe também que nem todo ótimo de Pareto está no "core".

Vejamos a seguir um modo de se dar sentido à conjectura de Edgeworth devido a Aumann. Antes de enunciarmos o teorema de Aumann, introduziremos um modelo para a economia de trocas ao qual a influência de um único agente na economia é insignificante. Para este modelo, mostraremos que cs conceitos de "core" o equilíbrio walrasiano são coincidentes, isto é, uma alocação $f$, está no "core" da economia se, e somente se, $f$ é uma alocação walrasiana.

Definição 1.14 Uma economia de trocas não atômica, $\varepsilon$, é uma aplicação mensurável definida em um espaço de medida não atomica $(A, B, \mu)$, com $\mu(A)=1$, tomando valores em $\mathcal{P} \times R_{+}^{n}$ tal que o vetor das dotaçồes iniciais dado por $\int \operatorname{proj}_{2} \circ \varepsilon \mathrm{d}_{\mu}$ é finito.

Observe que para cada $a \in A$, tendo $\varepsilon(a)=\left(\succ_{a}, e_{a}\right)$, temos que a preferência do agente $a$ é dada por $\operatorname{proj}_{1} \circ \varepsilon(a)$ e sua dotação inicial dada por $\operatorname{proj}_{2} \circ \varepsilon(a)$.

Definição 1.15 Uma alocação ou um estado para é é uma função integrável $f: A \rightarrow R_{+}^{n}$ tal que $\int f \mathrm{~d}_{\mu}=\int \operatorname{proj}_{2} \circ \varepsilon \mathrm{d}_{\mu}$. 
O conceito de economia de trocas não atômica, é na realidade, um conceito puramente abstrato. Sua interpretação pode ser a mesma dada no início da seção, pois qualquer economia $\varepsilon: A \rightarrow \mathcal{P} \times R_{+}^{n}$ pode ser vista como uma aplicação definida num espaço de medida $(A, B, \mu)$, tomando valores em $\mathcal{P} \times R_{+}^{n}$, onde $B$ é o conjunto de todos os subconjuntos do conjunto finito $A$ e a medida $u$ é a medide. de contagem normalizada, isto é, $\mu(E)=\frac{|E|}{|A|}$, onde $|E|$ é o número de elementos de $E$ e $|A|$ o número de elementos de $A$.

O número $\mu(E)$ é interpretado como a fração da totalidade dos agentes pertencentes à coalizão $E$.

A $\sigma$-álgebra $B$, que chamaremos $\sigma$-álgebra das coalizões, é introduzido por motivos técnicos. No caso de economias com um número finito de participantes, não há nenhuma restrição a possiveis coalizões.

Num espaço de medida não atômica $(A, B, \mu)$, o conjunto $A$ dos agentes econômicos é infinito e não enumerável.O conjunto $A$ será chamado de "continuun de agentes". E todo conjunto $S$ em $B$, será chamado de coalizão.

Observação: Dada uma alocação $f, f_{a}=f(a)$ denota uma nova cesta de bens para o agente. Dada uma coalizão $E$, e dada uma troca $f, \int_{E} f \mathrm{~d}_{\mu}$ representa a cesta média de bens alocados para os agentes de $E$.

Definição 1.16 Seja $f$ uma alocação para $\varepsilon$. Uma alocação $g$ domina ou melhora $f$ via coalizão $S \in B$, se:

a) $g(a) \succ_{a} f(a), \forall a \in S$;

b) $\mu(S)>0 e \int_{S} g \mathrm{~d}_{\mu}=\int_{S} e \mathrm{~d}_{\mu}$.

Evidentemente o conjunt.o de todas as alocações $f$ que não são dominadas ou melhoradas por qualquer alocação $g$, via qualquer coalizão $S \in B$ é chamado de "core" da economia. 
Definição 1.17 Uma alocação $f$ é chamada de alocação walrasiana se existir um vetor de preços $p \in R_{+}^{n}$ tal que:

a) Para todo $a \in A, f_{a}$ é maximal para $\succ_{a} \operatorname{em} \beta_{a}(p)=\left\{x \in X_{a} \mid p . x \leq p \cdot e(a)\right\}$;

b) $\int f \mathrm{~d}_{\mu}=\int e \mathrm{~d}_{\mu}$, isto é, a demanda de ben.s pelos agentes é totalmente suprida pelos bens ofertados pela economia.

O seguinte resultado é devido a Aumann (1964) "Markets with a continuum of traders", Econometrica 32:39-50.

Teorema 1.4 Seja $\varepsilon:(A, B, \mu) \rightarrow \mathcal{P}_{m o} \times R_{+}^{n}$ uma economia não atômica com $\int e \mathrm{~d}_{\mu} \gg 0$, então $\mathcal{W}(\varepsilon)=\ell(\varepsilon)$.

Prova: Vide Handbook of mathematical economics, vol. 2 , pag.843,844,845.

\subsection{Réplicas de uma economia e teorema limite}

Um outro modo de se dar sentido à conjectura de Edgworth é através da noção de réplica de uma economia e é devido a Debreu-Scarf.

Definição 1.18 Seja $\varepsilon$ uma economia de trocas possuindo m "traders". Uma n-réplica de $\varepsilon$, denotada por $\varepsilon^{(n)}$, consiste de n-cópias de $\varepsilon$ com mn "traders", consistindo na. reprodução de n-vezes as características de cada "trader", isto é, dado um "trader" j, $j=1, \ldots, m$, existem $n$ "traders" com as mesmas dotações iniciais do "trader" $j e$ mesma relação de preferência. Cada alocação em $\varepsilon$ define uma alocação em $\varepsilon^{(n)}$ por replicação, isto é, cada conjunto de n-"traders", com uma mesma característica, tem a mesma "commodity" como sua duplicata em $\varepsilon$. Se $X$ é uma alocação em $\varepsilon$, então $X^{(n)}$ denota a replicação da alocação em $X^{(n)}$. 
Teorema 1.5 (Teorema Limite (Debreu-Scarf )). Seja $\varepsilon$ uma economia de trocas com $m$ "traders". Se cada "agente" tem funçâo utilidade fortemente monotônica (isto é, estritamente crescente), fortemente convexa, e contínua, e cada "trader" tem dotaçôes iniciais estritamente positivas, então uma alocação $X$ é uma alocação competitiva para $\varepsilon$ se, e somente se, $X^{(n)}$ está no "core" le $\varepsilon^{(n)}$ para todo $n \geq 1$.

Prova: Vide "A Limit Theorem on the core of an Economy" (1963) International Economic Review, 4, 235-246. 


\section{Capítulo 2}

\section{Economias de trocas não "standard"}

Neste capítulo procuraremos dar sentido a conjectura de Edgeworth usando como ferrameta matemática a análise não "stanciard". Definiremos um modelo para economia de trocas que chamaremos de economia de trocas não "standard", redefiniremos os conceitos de equilíbrio competitivo ou "Walrasiano" e de "core" ou núcleo econômico. Este capítulo esta dividido em duas seções; o primeiro trará como principal resultado a equivalência do "core" e o equilibrio competitivo para economias de trocas não "standard", a segunda seção trata de réplicas econômicas e uma versão não "standard" para o teorema limite de Debreu- Scarf. Para os não iniciados em análise não "standard" recomendamos uma leitura prévia do apêndice C, neste apêndice serão encontrados os principais teoremas e conceitos utilizados neste capítulo. Afim de não desviar a atencão do leitor, os resultados sobre convexidade, num contexto não "standard" para economia de trocas não standard, estão dispostos no apêndice B.

Antes de definirmos um modelo para economia de trocas em um contexto não "standard" introduziremos as seguintes notações:

a) $\forall x \in^{*} R^{n}$, a mônada de $x, \mu(x)$, é o conjunto de todos os pontos de ${ }^{*} R^{n}$ cuja distância a $x$ é infinitesimal. Se $y \in \mu(x)$ escreveremos $x \approx y$ (le-se $x$ infinitamente próximo de $y$ ). 
b) $\forall x \in^{*} R^{n}, x \geq y$ significa $x_{i} \geq y_{i}$ para todo $i$,

$x>y$ significa $x_{i} \geq y_{i}$ para todo $i$, e $x_{i}>y_{i}$ para algum $i$,

$x \gg y$ significa $x_{i}>y_{i}$ para todo $i$,

$x \gtrsim y$ significa $x \geq y$ ou $x \approx y$.

\subsection{A equivalência entre o "core" e o equilíbrio Wal- rasiano}

Definição 2.1 Uma economia de trocas não "standard", $\varepsilon$, é dada por um par de funções internas ${ }^{*} I,{ }^{*} P$, definidas em um segmento inicial $T \subset{ }^{*} N$, isto $e ́, T=\{1,2, \ldots, \omega\}$, tal que $|T|=\omega \in{ }^{*} N \backslash N$, tomando valores em ${ }^{*} \Omega_{n}$ e $\mathcal{P}\left({ }^{*} \Omega_{n} \times{ }^{*} \Omega_{n}\right)$, respectivamente, onde $n$ é um natural standard. ${ }^{*} \Omega_{n}=\left\{x \in{ }^{*} R^{n}: x_{i} \geq 0, \forall i\right\}$ que chamaremos de ortante positivo $d e^{*} R^{n}$.

i) A função ${ }^{*} I$ associa a cada consumidor $t \in T$, a sua dotação inicial, ou cesta de "commodities" inicial, ${ }^{*} I(t)=x_{i} \in{ }^{*} \Omega_{n}$.

ii) $I(t)$ é "standard" limitada, isto é, existe um vetor $r \in \Omega_{n} \subset{ }^{*} \Omega_{n}$ tal que $I(t) \leq r$, $\forall t$.

iii) $\frac{1}{\omega} \sum_{t=1}^{\omega} I(t) \not{\approx} 0$, isto é, a cesta média de "commodities" da economia é não nula, a média de nenhum bem é infinitesimal e grande parte dos consumidores possuem quantidades não infinitesimais dos bens disponíveis para consumo.

Observação: A operação $\Sigma$ que foi utilizada acima é obtida por transferência, do seguinte modo: Sejam $\Omega_{n}$ e $\mathcal{P}_{\text {Fin }}\left(\Omega_{n}\right)$, onde $\mathcal{P}_{\text {Fin }}\left(\Omega_{n}\right)$ denota o conjunto das partes finitas de $\Omega_{n}$. Defina:

$$
\Sigma: \mathcal{P}_{\text {Fin }}\left(\Omega_{n}\right) \rightarrow \Omega_{n}
$$




$$
\left\{a_{1}, \ldots, a_{n}\right\} \mapsto \frac{1}{k} \sum_{j=1}^{k} a_{j}
$$

aplicando o monomorfismo ${ }^{*}$ obtemos: ${ }^{*} \Sigma:{ }^{*} \mathcal{P}_{\text {Fin }}\left(\Omega_{n}\right) \rightarrow{ }^{*} \Omega_{n}$, onde ${ }^{*} \Sigma$ é extensão de $\Sigma$, e t,al que se $\left\{a_{1}, \ldots, a_{k}\right\} \in \mathcal{P}_{\text {Fin }}\left(\Omega_{n}\right)$,

$$
\frac{1}{k} \sum_{j=1}^{k} a_{j}=\frac{1}{k}{ }^{*} \sum_{j=1}^{k}{ }^{*} a_{j} .
$$

Continuaremos a utilizar o símbolo $\Sigma$ para indicar a operação ${ }^{*} \Sigma$, ficando claro que $\Sigma$ é obtida como descrito acima.

iv) Relembramos que a relação, $Q$, onde $Q=\left\{\left\langle t,{ }^{*} P(t)=\succ_{t}\right\rangle: t \in T, \succ_{t} \subseteq{ }^{*} \Omega_{n} \times{ }^{*} \Omega_{n}\right\}$ é interna.

Para todo t,

$\alpha) \succ_{t}$ é irreflexiva, isto é, $x \succ_{t} y \Rightarrow x \neq y$;

$\beta)$ Se $x \geq y$, então $x \succ_{t} y$, isto é, $\succ_{t}$ é monotômica;

$\gamma$ ) Para todo $x, y \in{ }^{*} \Omega_{n}, x, y$ finitos, se $z \approx 0$, então para todo $u \approx x+y, z \approx y$, $w \succ z$

$\left.\gamma^{\prime}\right) \succ_{t}$ é tal que se $x \approx y$ e $x \succ_{t} y$ existe $\delta>0, \delta$ "standard" tal que, para todo $z \in B(x, \delta)$, a bola de centro $x$ e raio $\delta$, tem-se $z \succ_{1} y$.

\section{Proposição 2.1 São equivalentes:}

i) Se $x \succ_{t} y$ e $z \in \mu(x)$, então $z \succ_{t} y$;

ii) $\succ_{t}$ é tal que se $x \not \approx y, x \succ_{t} y \Rightarrow \Xi \delta>0, \delta \in R: \forall z \in B(x, \delta) \Rightarrow z \succ_{t} y$.

Prova: $\Rightarrow)$ Suponha $x \not \approx y, x \succ_{t} y\left((\forall z \in \mu(x)) \Rightarrow z \succ_{t} y\right)$ afirmamos que $\succ_{t}$ localmente não saciada. Seja $\Delta=\left\{\delta \in{ }^{*} R: \forall z(|z-x|<\delta) \Rightarrow z \succ, y\right\}$. É claro que $m(0) \subseteq \Delta$, onde $m(0)$ representa a mônada de $0 \in{ }^{*} R$.

Afirmamos que $\Delta$ é interno, pois como 
a) ${ }^{*} R_{+}$é interno;

b) a prorpriedade $\forall z(|z-x|<\delta) \Rightarrow z \succ_{\iota}, y$, envolve um número finito de funções internas a saber:

b", | | : ${ }^{*} R^{n} \rightarrow{ }^{*} R$, obtida pela transferência da norma usual em $R^{n}$. (Obs.: usaremos também a notação * $\rho$, quando conveniente, para indicar a extensão da norma usual em $R^{n}$.)

b") $<$, obtida por transferncia da ordem usual em $R$;

b"') $\succ_{t}$ por hipótese.

Logo $\Delta$ satisfaz as hipóteses do teorema de Keisler e portanto interno. Portanto pelo princípio do esparramamento temos.

c) $\exists \delta>0(\delta \in R) \quad \forall z(|z-x|<0) \Rightarrow z \succ_{t} y$.

Agora suponha $\succ_{t}$ localmente não saciada, $x \not z y, x \succ_{t} y$, afirmamos que $(\forall z \in$ $\left.\mu(x), z \succ_{t} y\right)$.

Prova: Imediata.

Funçes de trocas

Definição 2.2 Chamaremos de trocas, ou funçâo de trocas, as funções internas $Y, Z, \ldots$, $X: T \rightarrow{ }^{*} \Omega_{n}$, que associa a cada t uma nova cesta de "commodities" $X(t)$.

Definição 2.3 Chamaremos alocação final, ou simplesmente alocaçâo, a função de troca $Y$ com as seguintes propriedades:

a) $Y$ é "standard" limitada.

b) $\frac{1}{\omega} \sum_{t=1}^{\omega} Y(t) \approx \frac{1}{\omega} \sum_{t=1}^{\omega} I(t)$. 
v) A condiçâo iv) e o teorema de Kiesler, implicam que para toda funçâo interna $X, Y, \ldots, Z \in{ }^{*} \Omega_{n}^{T}$, que o conjunto $\{t: X(t) \succ, Y(t)\}$ é interno.

\section{Coalizâo}

Definição 2.4 Uma coalizào, S, é qualquer subconjunto interno de T. Deve-se entender coalizão como um conjunto de consumidores que se unem, e de forma cooperativa tentarào impor uma alocação na economia que maximize suas preferências.

Definição 2.5 Diz-se que uma coalizão, $S$, é negligenciável se $\frac{|S|}{\omega} \approx 0$, isto é, $S$ é uma coalizão negligenciável se o número de elementos de $S$ for pequeno perante todos os consumidores da economia.

\section{Alocações e coalizões}

Lema 2.1 Se $S$ é negligenciável, entâo para toda alocaçầo $X$ temos $\frac{1}{\omega} \sum_{t \in S} X(t) \approx 0$.

Prova: Seja $X$ uma alocação, temos que existe $b \in{ }^{*} \Omega_{n}$ "standard" tal que $X(t) \leq b$ para todo $t$ em $S$; vem daíque $0 \leq \frac{1}{\omega} \sum_{t \in S} X(t) \leq \frac{1}{\omega} \sum_{t \in S} b=\frac{|S|}{\omega} b \approx 0$.

Se $S$ é negligenciável, os ganhos obtidos pelos consumidores $t \in S$, são desprezíveis perante a economia.

Definição 2.6 Diz-se que uma coalizão, S, é factivel para uma alocação $Y$ se:

$$
\frac{1}{\omega} \sum_{t \in S} Y(t) \approx \frac{1}{\omega} \sum_{t \in S} I(t) .
$$

Definição 2.7 Diz-se que uma alocação $Y$ domina uma alocação $X$ via coalizão $S$, se:

a) $S$ é factivel para a alocação $Y$; 
b) $\forall t \in S(Y(t) \not X X(t))\left(\forall z \in \mu(Y(t)) \Rightarrow z \succ_{t} X(t)\right.$.

(Obs.: sendo $\succ$, localmente nào saciada temos, pela proposição 1 acima, que $\exists \delta>0$ $(\delta \in R)\left(\forall z \in B(Y(t), \delta) \Rightarrow z \succ_{t} X(t).\right)$

"Core"

Definição 2.8 Chama-se "core" de $\varepsilon . \ell(\varepsilon)$, ao conjunto de todas as alocaçòes $X$ que nầo são dominadas por qualquer alocação $Y$ via coalizâo $S$. O "core" é portanto o conjunto das alocaçôes que bloqueiam ganhos por coalizão.

Sistema de preços, e o budget set

Definição 2.9 Um vetor de preços p, é um vetor não "standard" finito pertencente a * $\Omega_{n}$ tal que $p \gg 0$. Cada coordenada $p_{i}$ de p determina o preço de uma unidade do bem $x_{i}$ de uma cesta de "commodities" x. O valor de uma cesta de "commodities" $x$ é dado pelo produto interno de p.x, o produto internı é obtido por extensão do produto interno usual $\mathrm{em}{ }^{*} R^{n}$.

Restrição orçamentária (Budget set)

Definição 2.10 Para cada sistema de preços $p$, o "budget set" do consumidor t é o conjunto

$$
B_{p}(t)=\left\{x \in{ }^{*} \Omega_{n}: p . x \leq p . I(t)\right\} .
$$

Diz-se que uma cesta de "commodities" y é maximal em $B_{p}(t)$ se

$$
y \in B_{p}(t), \nexists x \in B_{p}(t)(x \not z y)(\forall z \in \mu(x)) \Rightarrow z \succ_{t} y .
$$

Equilíbrio competitivo ou equilíbrio Walrasiano 
Definição 2.11 O equilíbrio competitivo ou Walrasiano, w( $(\varepsilon)$, é um par $(p, X)$ tal que $p$ é um vetor de preços e $X$ uma alocaçâo, e existe um subconjunto interno $K \subset A=\{t \in$ $T: X(t)$ é maximal em $\left.B_{p}(t)\right\}$ tal que $\frac{|K|}{\omega} \approx 1$.

O equilíbrio walrasiano é portanto um sistema de preços e uma alocação que maximiza a preferência de quase todos os consumidores da economia.

\section{Conjunto pleno de consumidores}

Definição 2.12 Seja ع uma economia, não "standard", de trocas onde o conjunto de consumidores $T$ é interno, e $|T|=\omega$ inteiro positivo infinito. Um conjunto de consumidores $U$ é dito pleno, se $U$ é interno e $\frac{|T-U|}{\omega} \approx 0$.

Seja $X(t)$ uma alocação no "core" de $\varepsilon$ fixada. Considere os conjuntos

$$
\begin{aligned}
F_{k}(t) & =\bigcup_{q \in N}\left\{x \in{ }^{*} \Omega_{n}:|x|<q,\left(\forall w \in{ }^{*} \Omega_{n}\left({ }^{*} p(x, w)<\frac{1}{k}\right) w \succ_{1} X(t)\right\} \quad \mathrm{e}\right. \\
G_{k}(t) & =F_{k}(t)-I(t) .
\end{aligned}
$$

Sejam $G(t)=\bigcup_{k \in N} G_{k}(t), F(t)=\bigcup_{k \in N} F_{k}(t)$, temos $G(t)$ e $F(t) S$-abertos.

Prova: basta provar que $F_{k}(t)$ é $S$-aberto.

Seja $x \in F(t)$, temos então que $x \in F_{k}(t)$ para algum $k$. Seja $\delta$ real "standard" tal que $\delta<\frac{1}{2 k}$, e considere a $S$-bola, $S(x, \delta)$, temos para todo $y \in S(x, \delta)$, para todo $w \in{ }^{*} \Omega_{n}$ tal que ${ }^{*} \rho(y, w)<\frac{1}{2 k}$, tem-se que

$$
{ }^{*} \rho(x, w) \leq{ }^{*} \rho(x, y)+{ }^{*} \rho(y, w)<\frac{1}{2 k}+\frac{1}{2 k}=\frac{1}{k} \Rightarrow w \succ_{1} X(t)
$$

e portanto a $S$-bola, $S(x, \delta) \subset F_{k}(t) \subset F(t)$, logo o conjunto $F(t)$ é $S$-aberto, e conseqüentemente $G(t)$ aberto.

Seja $\Delta(U)$ o "S-convex hull" da união $\bigcup_{t \in U} G(t)$.

Lema 2.2 (Lema Principal) Existe um conjunto pleno de consumidores, $U$, tal que $0 \notin$ $S-\operatorname{int}(\Delta(U))$. 
Prova: Para cada $x \in{ }^{*} R^{n}$, seja $G^{-1}(x)=\{t \in T: x \in G(t)\}$, temos então que $G^{-1}(x)=$ $\bigcup_{k \in N} G_{k}^{-1}(x)$, em que $G_{k}^{-1}(x)=\left\{t \in T:\left(\forall \cdot u \in{ }^{*} \Omega_{n}{ }^{*} p(x+I(t), w)<\frac{1}{k}\right) w \succ_{1} X(t) \mathrm{e}\right.$ $\left.x+I(t) \in{ }^{*} \Omega_{n}\right\}$.

Prova: Suponha $t \in G^{-1}(x)$, temos $x \in G(t) \Rightarrow x \in G_{k}(t)=F_{k}(t)-I(t)$ para algum $k \in$ $N$, e portanto $x+I(t) \in{ }^{*} \Omega_{n},\left(\forall w \in{ }^{*} \Omega_{n},{ }^{*} \rho(w, x+I(t))<\frac{1}{k}\right) w \succ, X(t) \Rightarrow t \in G_{k}^{-1}(x)$. Logo,

$$
G^{-1}(x) \subseteq \bigcup_{k \in N} G_{k}^{-1}(t)
$$

Agora, suponha $t \in \bigcup_{k \in N} G_{k}^{-1}(x)$, temos $t \in G_{k}^{-1}(x)$ para algum $k \in N$, e portanto $\left(\forall w \in{ }^{*} \Omega_{n},{ }^{*} \rho(w, x+I(t))<\frac{1}{k}\right) w \succ_{t} x(t) \Rightarrow x \in G(t), \log 0$

$$
\bigcup_{k \in N} G_{k}^{-1}(x) \subseteq G^{-1}(x)
$$

o que prova a afirmação.

Segue de (v) que para todo $k \in N$ e todo $x \in{ }^{*} \Omega_{n}, G_{k}^{-1}(x)$ é intcrno, e portanto $G^{-1}(x)$ é interno.

Seja $L$ o conjunto de todos os racionais "standard", $r, r \in{ }^{*} R$ tal que para todo $k \in N$, $G_{k}^{-1}(r)$ é negligenciável. Seja $r_{1}, \ldots, r_{m}, \ldots$ uma enumeração desses racionais. Afirmamos que para todo $r_{i}$ existe um $v_{i} \in{ }^{*} N$ tal que $G^{-1}\left(r_{i}\right) \subseteq G_{v_{i}}^{-1}\left(r_{i}\right)$, onde $G_{v_{i}}^{-1}\left(r_{i}\right)$ é interno e negligenciável.

Prova: Primeiramente, temos que para todo $k, G_{k}^{-1}(x) \subset G_{k+1}^{-1}(x)$, pois para todo $t \in$ $G_{k}^{-1}(x) \Rightarrow x \in G_{k}^{-1}(t) \supset G_{k+1}^{-1}(t)$, pois basta observar que para todo $x \in G_{k+1}^{-1}(t)$ tem-se que $\left(\forall w \in{ }^{*} \Omega_{n},{ }^{*} p(w, x+I(t))<\frac{1}{k+1}<\frac{1}{k}\right) w \succ_{t} X(t) \Rightarrow x \in G_{k}^{-1}(t)$ e portanto para todo $t \in G_{k}^{-1}(x) \Rightarrow t \in G_{k+1}^{-1}(x)$.

Defina

$$
\begin{aligned}
\varphi: N & \rightarrow{ }^{*} V_{m}(R) \\
k & \mapsto G_{k}^{-1}(x),
\end{aligned}
$$


por abrangncia temos:

$$
\begin{aligned}
{ }^{*} \varphi:{ }^{*} N & \rightarrow{ }^{*} V_{m}(R) \\
k & \mapsto G_{k}^{-1}(x) .
\end{aligned}
$$

Agora, seja

$$
\begin{aligned}
\Gamma= & \left\{k \in{ }^{*} N: \forall j \in{ }^{*} N\left(j<k{ }^{*} \varphi(j) \subseteq{ }^{*} \varphi(j+1) \subseteq{ }^{*} \varphi(k) \text { e } \quad \frac{1}{\omega}\left|{ }^{*} \varphi(j)\right|<\frac{1}{j},\right.\right. \\
& \left.\operatorname{com}^{*} \varphi(k) \subseteq T,\right\}
\end{aligned}
$$

onde $T$ conjunto dos consumidores. Temos $\Gamma$ conjunto interno e contém todos os naturais "standard", logo por esparramamento temos que existe $k$ hiperfinito tal que ${ }^{*} \varphi(j) \subseteq$ ${ }^{*} \varphi(k)$ para todo $j \in N$ e portanto $\exists v_{i} \in{ }^{*} N: G^{-1}\left(r_{i}\right) \subseteq G_{v_{i}}^{-1}\left(r_{i}\right)$.

Seja $B_{k}=\bigcup_{i=1}^{k} G_{v_{i}}^{-1}\left(r_{i}\right), k \in N$, com o mesmo argumento utilizado acima podemos obter $\rho \in{ }^{*} N$ tal que $B_{k} \subset B_{\rho}$, para todo $k \in N$, com $B_{\rho}$ negligenciável.

Seja $U=T-B_{\rho}$, temos $U$ subconjunto pleno de $T$, isto é, $\frac{\left|T-B_{\rho}\right|}{\rho} \approx 1$. Agora, suponha $0 \in S-\operatorname{int}(\Delta(U))$. Então existe $x \in{ }^{*} R^{n}, x \not{\approx} 0$ tal que $-x \in \Delta(U)$. Pela definição de $\Delta(U),-x$ é combinação convexa de $k$ pontos pertencentes a $\bigcup_{\substack{t \in\{1, \ldots, k \\ t \in(1, \ldots}} G\left(t_{i}\right)$, em que $k$ finito. Isto é, existem $t_{1}, \ldots, t_{k} \in U$ (não necessariamente distintos), pontos $x_{i} \in G\left(t_{i}\right)$, $i=1, \ldots, k$; números reais "standards" $\beta_{1}, \ldots, \beta_{k}$, tais que $\sum_{t=1}^{k} \beta_{i} x_{i}=-x \not{\nsim} 0$, com $\beta_{j}>0$.

De $x_{i} \in G\left(t_{i}\right)$ temos para algum $q \in N\left(\forall w \in{ }^{*} R^{n}:{ }^{*} p\left(x_{i}+I\left(t_{i}\right), w\right)<\frac{1}{q}\right) w \succ_{t} X\left(t_{i}\right)$. Sendo $G\left(t_{i}\right)$ aberto para todo $i$, e portanto existem pontos racionais "stantards" $r_{i} \in G\left(t_{i}\right)$ suficientemente próximos de $x_{i}$ e existem números racionais "st,andards" $\gamma_{i}$ suficientemente próximos de $\beta_{i}$ para que se tenha $\sum_{i=1}^{k} \gamma_{i} r_{i} \not \approx 0$ e $\sum_{i=1}^{k} \gamma_{i}=1$.

Seja $-r=\sum_{i=1}^{k} \gamma_{i} r_{i}$ e tome um consumidor arbitrário $t_{0} \in U$. Vist.o que $r \not \approx 0$, temos $\alpha x+I\left(t_{0}\right) \stackrel{\gg}{\not} X\left(t_{0}\right)$, para $\alpha$ racional positivo suficientemente grande. Portanto, pela monotonicidade da relação de preferência, assumida para todos os consumidores, temos $\alpha r+I\left(t_{0}\right) \succ \succ_{t_{0}} X\left(t_{0}\right)$; isto é, $\forall w \in \mu\left(\alpha r+I\left(t_{0}\right)\right)$ tem-se $w \succ_{t_{0}} X\left(t_{0}\right)$. 
Seja $\Psi=\left\{\left(\delta \in{ }^{*} R\right)\left(\forall u \in{ }^{*} \Omega_{n}{ }^{*} \rho\left(w, \alpha r+I\left(t_{0}\right)\right)<\delta\right) \Rightarrow u^{\prime} \succ_{1}, \mathrm{Y}\left(t_{0}\right)\right\}$. Temos $m(0) \subset \Psi, \Psi$ é interno e por esparramamento existe $\delta>0, \delta \in R$ e portanto existe $k \in N$ tal que $\frac{1}{k}<\delta$ e $\left(\forall w \in{ }^{*} \Omega_{n}\right)\left({ }^{*} \rho\left(w, \alpha r+I\left(t_{0}\right)\right)<\frac{1}{k}<\delta\right) \Rightarrow \iota^{\prime} \succ_{\iota_{0}} X\left(t_{0}\right) ; \log \alpha a r \in G\left(t_{0}\right)$.

Agora, fixe $r_{0}=\alpha r, \alpha_{0}=\frac{1}{\alpha+1}, \alpha_{t}=\frac{\alpha \gamma_{1}}{\alpha+1}$, para $i=1, \ldots, k$. Temos cutão $\alpha_{i}>0$ para todo i. e

$$
\sum_{i=0}^{k} \alpha_{i}=\alpha_{0}+\sum_{i=1}^{k} \alpha_{i}=\frac{1}{\alpha+1}+\frac{a}{\alpha+1} \sum_{i=1}^{k} \gamma_{i}=1
$$

Entretanto,

$$
\sum_{i=0}^{k} \alpha_{i} r_{i}=\alpha_{0} r_{0}+\sum_{i=1}^{k} \alpha_{i} r_{i}=\frac{1}{\alpha+1} \alpha r+\sum_{i=1}^{k} \frac{\alpha}{\alpha+1} \gamma_{i} r_{i}=\frac{a}{\alpha+1}(r-r)=0
$$

e para todo $i, r_{i} \in G\left(t_{i}\right)$. Então, $t_{i} \in G^{-1}\left(r_{i}\right)$, e como $t_{i} \in U$, segue que $r_{i} \notin L$.

Portanto, para todo $i, \exists m_{i} \in N: \frac{\left|G_{m_{i}}^{-1}\left(r_{i}\right)\right|}{\omega} \not \approx 0$.

Vamos mostrar agora que para um $\delta$ "standard" positivo (suficientemente pequeno), podemos encontrar subconjuntos disjuntos $A_{i} \subset G_{m_{i}}^{-1}\left(r_{i}\right)$ tal que $\frac{\left|A_{1}\right|}{\omega} \approx \delta a_{i}$.

$$
\begin{gathered}
\text { Sejam } \delta=\left(\frac{|B|}{\omega}\right), \text { onde } B=G_{m_{i}}^{-1}\left(r_{i}\right) ; A_{0}=\left\{t_{1}, \ldots, t_{\left.\mid \delta \omega \alpha_{0}\right]}\right\},|B| \\
A_{1}=\left\{t^{\cdot}\left(\left[\delta \omega \alpha_{0}\right]+1\right), \ldots, t_{\left.\left(\left[\delta \omega \alpha_{0}\right]+\left|\delta \omega \alpha_{1}\right|\right)\right\}, \ldots,}\right. \\
A_{k}=\left\{t^{t}\left(\sum_{j=0}^{k \cdots 1}\left[\delta \omega \alpha_{j} \mid+1\right), \ldots, t_{L}\right\},\right.
\end{gathered}
$$

onde $\left[\delta \omega \alpha_{j}\right]=\max \left\{n \in N ; n<\delta \omega \alpha_{j}\right\}, j=0, \ldots, k-1$.

(Observe que $\sum_{j=0}^{k-1}\left[\delta \omega \alpha_{j}\right]<L$, pois $\left[\delta \omega \alpha_{j}\right]+\frac{1}{k}<\delta \omega \alpha_{j}+\frac{1}{k}$, e portanto $\sum_{j=0}^{k-1}\left[\delta \omega \alpha_{j}\right]+1<$ $\delta \omega\left(1-\alpha_{k}\right)+1$ e como $\delta \omega\left(1-\alpha_{k}\right)+1<L$ (por construção), temos $\delta\left(1-\alpha_{k}\right)+\frac{1}{\omega}<\frac{L}{\omega} \mathrm{e}$ portanto ${ }^{0}\left(\delta\left(1-\alpha_{k}\right)+1\right)<{ }^{0}\left(\frac{L}{\omega}\right)$, isto é, $\delta\left(1-\alpha_{k}\right)<\delta$. $)$

Seja $A=\bigcup_{t=0}^{k} A_{i}$ e defina a seguinte função interna:

$$
Y(t)=r_{i}+I(t), t \in A_{t} \quad \text { e } \quad Y(t)=I(t), t \notin A
$$


É claro que $Y(t) \in{ }^{*} \Omega_{n}, \forall t \in A, \forall t \in A_{t}$. Segue que $r_{i} \in G(t)$, isto é, $A_{i} \subset G_{m_{i}}^{-1}\left(r_{i}\right)$. Portanto, $r_{i}+I(t) \in F_{m_{i}}(t) \subset{ }^{*} \Omega_{n}$. Agora,

$$
\begin{aligned}
\frac{1}{\omega} \sum_{t \in A} Y(t) & =\frac{1}{\omega} \sum_{i=0}^{k} \sum_{t \in A_{i}} Y(t)=\frac{1}{\omega} \sum_{i=0}^{k} \sum_{t \in A_{i}}\left\{r_{i}+I(t)\right\} \\
& =\frac{1}{\omega} \sum_{i=0}^{k} \sum_{t \in A_{i}} r_{i}+\frac{1}{\omega} \sum_{i=0}^{k} \sum_{t \in A_{i}} I(t) \\
& =\frac{1}{\omega} \sum_{i=0}^{k}\left|A_{i}\right| r_{i}+\frac{1}{\omega} \sum_{t \in A_{i}} I(t) \approx \delta \sum_{i=0}^{k} \alpha_{i} r_{i}+\frac{1}{\omega} \sum_{t \in A} I(t) \approx 0+\frac{1}{\omega} \sum_{t \in A} I(t) \\
& =\frac{1}{\omega} \sum_{t \in A} I(t),
\end{aligned}
$$

e portanto $A$ é factivel para $Y$. Dado que $Y(t)=I(t)$ para $t \notin A$, temos que $Y$ é uma alocação. Finalmente, $A_{i} \subset G_{m_{i}}^{-1}\left(r_{i}\right)$, segue que $r_{i}+I(t) \succ_{t} X(t)$ para todo $t \in A_{i}$, isto é, $Y(t) \succ_{t} X(t)$ para todo $t \in A$. Logo, $A$ é não negligenciável, e portanto $X$ não está no "core"; contra hipótese.

Teorema 2.1 Se e é uma economia de trocas não "standard" satisfazendo as propriedades listadas anteriormente, então uma funçâo de trocas $X$ está no "core" de $\varepsilon$ se, e somente se, existe um vetor de preços p tal que o par $(p, X)$ é um equilíbrio walrariano.

Prova: $w(\varepsilon) \subset \ell(\varepsilon)$.

Seja $(p, X)$ equilíbrio walrasiano para $\varepsilon$, isto é, existe um conjunto interno $K$, tal que $\frac{|K|}{\omega} \approx 1$ e $X(t)$ é maximal em $B_{p}(t)$ para todo $t \in K$. Se $X(t) \notin \ell(\varepsilon)$, então existe uma alocação $Y(t)$ e uma coalizão não negligenciável $S$, tal que $\frac{1}{\omega} \sum_{t \in S} Y(t) \approx \frac{1}{\omega} \sum_{t \in S} I(t)$ e para todo $t \in S, Y(t) \succ_{t} X(t)$ e $Y(t) \not \approx X(t)$.

Seja $M=S \cap K$, temos

i) $M$ interno ( teorema de Kiesler),

ii) $\frac{|M|}{\omega} \approx \frac{|S|}{\omega}$. 
Prova: temos $0 \leq\left|S \cup K^{\prime}\right| \leq|T|=\omega \Rightarrow\left|S \cup K^{*}\right|=|S|+|K|-|S \cap K| \leq \omega$. dividindo-se ambos os menbros da desigualdade por $\omega$, vem:

$$
\frac{|S|}{\omega}+\frac{|K|}{\omega}-\frac{|M|}{\omega} \leq 1
$$

e sendo $|M| \leq|S|$, temos

$$
0 \leq \frac{|S|}{\omega}-\frac{|M|}{\omega} \leq 1-\frac{|K|}{\omega} \approx 0 \Rightarrow \frac{|S|}{\omega} \approx \frac{|M|}{\omega}
$$

iii) para todo $t \in M, Y(t) \succ, X(t)$.

Prova: imediato pois $M \subseteq S$.

iv) $\forall t \in M, p . Y(t) \not{\not} p . I(t)$.

Prova: Suponha que $p . Y(t) \lesssim p . I(t) \Rightarrow Y(t) \in B_{p}(t), \forall t \in M$. Como $Y$ domina $X$ via coalizão $S$, temos $\forall z \in \mu(Y(t))\left(z \succ_{t} X(t)\right)$, absurdo pois $X(t) \not \forall Y(t)$ e $X(t)$ maximal em $B_{p}(t)$.

Agora, de iv) temos que para todo $t \in R, p .(Y(t)-I(t)) \not{\not} 0$.

Sendo $T$ hiperfinito, $M \subset T, M$ interno, vem que existe

$$
t_{0} \in M I\left(\forall t \in M, p \cdot\left(Y(t)-I(t) \geq p \cdot\left(Y\left(t_{0}\right)-I\left(t_{0}\right)\right) .\right.\right.
$$

Mas p. $\left(Y\left(t_{0}\right)-I\left(t_{0}\right)\right) \not{\approx} 0$. Tome $\lambda=\frac{1}{2} p .\left(Y\left(t_{0}\right)-I\left(t_{0}\right)\right)$, temos ent.ão que

$$
p .(Y(t)-I(t)) \geq p .\left(Y\left(t_{0}\right)-I\left(t_{0}\right) \not{\approx} \lambda \ddot{\approx} 0 \text {, para todo } t \in M,\right.
$$

conseqëntemente, para todo $t \in M$,

$$
\begin{aligned}
& \frac{1}{\omega} \sum_{t \in R} p(Y(t)-I(t)) \geq \frac{1}{\omega} \sum_{t \in R} p\left(Y\left(t_{0}\right)-I\left(t_{0}\right)\right)=\frac{|M|}{\omega} p \cdot\left(Y\left(t_{0}\right)-I\left(t_{0}\right)\right) \geq \frac{|M|}{\omega \mid} \lambda \gtrsim 0 \\
& \Rightarrow p \cdot \frac{1}{\omega} \sum_{t \in R} \frac{Y(t)}{\omega}=\frac{1}{\omega} \sum_{i \in \omega} p . Y(t) \not{\approx} p \cdot \sum_{t \in R} \frac{I(t)}{\omega} .
\end{aligned}
$$


o que contradiz o fato de $S$ ser factível para $Y$. Pois, sendo $S$ factível para a alocação $Y$, temos:

$$
\frac{1}{\omega} \sum_{t \in S} Y(t)=\frac{1}{\omega} \sum_{t \in M} Y(t)+\frac{1}{\omega} \sum_{t \in(S-M)} Y(t) \approx \frac{1}{\omega} \sum_{t \in M^{\prime}} I(t)+\frac{1}{\omega} \sum_{t \in(S-M)} I(t)=\frac{1}{\omega} \sum_{t \in S} I(t) .
$$

$\operatorname{Mas} \frac{|S|}{\omega} \approx \frac{|M|}{\omega}$

$$
\begin{aligned}
0 & \approx \frac{1}{\omega} \sum_{t \in S} Y(t)-\frac{1}{\omega} \sum_{t \in M} Y(t)=\frac{1}{\omega} \sum_{t \in(S-M)} Y(t) \\
& \approx \frac{1}{\omega} \sum_{t \in(S-M)} Y(t)=\frac{1}{\omega} \sum_{t \in S} I(t)-\frac{1}{\omega} \sum_{i \in M} I(t) \approx 0
\end{aligned}
$$

e portanto $p . \sum_{t \in M} \frac{Y(t)}{\omega} \approx p . \sum_{t \in M} \frac{I(t)}{\omega}$. o que contradiz iv). Logo $w(\varepsilon) \subseteq \ell(\varepsilon)$.

Vamos mostrar agora que $\ell(\varepsilon) \subseteq w(\varepsilon)$.

Suponha $X$ no "core" de $\varepsilon$ e seja $U$ conjunto pleno de consumidores como no lema principal. Temos pelo lema principal que $0 \notin S-\operatorname{int}(\Delta(U))$; e portanto pelo lema da separação existe um vetor "standard" $p \neq 0$ tal que para todo $x \in S-\operatorname{int}(\Delta(U))$, $p . x \gtrsim 0$. Portanto para todo $y \in G(t), p . y \gtrsim 0$, onde $S-\operatorname{int}(G(t))=G(t)$, ist,o porque o $S-\operatorname{int}(\Delta(U))=$ "S-convex hull" de $G(t)$. O que é equivalente a dizer que $p . x \gtrsim p . I(t)$, pois dado $y \in G(t)$, temos $y=x-I(t)$, onde $x \in F(t)$, dado que $p \cdot y \gtrsim 0$, vem que $p \cdot y=p \cdot(x(I(t)) \gtrsim 0 \Rightarrow p \cdot x \gtrsim I(t)$. Agora, se $x \in F(t) \operatorname{com} p \cdot y \gtrsim p \cdot I(t)$, temos $p .(x-I(t)) \gtrsim 0$, como $x \in F(t)$ vem que $x-I(t) \in G(t)$.

Suponha $z \in \Omega_{n} \subset{ }^{*} \Omega_{n}$, isto é, $z$ vetor "standard" com $z \geq 0$. Então $z+X(t) \in F(t)$ para todo $t \in T$, pois temos que $X(t)$ e $z \in{ }^{*} \Omega_{n}$, e por iv) e $\gamma$ ) vem que para todo $w \approx z+X(t), w \succ_{t} X(t)$.

Definindo $\Theta=\left\{\left(\delta \in{ }^{*} R\right):\left(\forall w \in{ }^{*} \Omega_{n}{ }^{*} \rho(w, x+X(t))<\delta\right) w \succ_{1} X(t)\right\}$, temos $\Theta$ interno e contém todos os infinitésimos e por esparramamento temos que existe $\delta>0$ $(\delta \in R)$ e portanto existe $k \in N$ tal que $\frac{1}{k}<\delta$, e $\left(\forall w \in{ }^{*} \Omega_{n}{ }^{*} \rho(w, z+X(t))<\frac{1}{k}<\delta\right) w \succ_{t}$ $X(t)$ e portanto $z+X(t) \in F(t)$.

Afirmamos que $p \geq 0$. 
Suponha que para algum $i, \rho_{i}, 0 \in R$, podemos escolher $z \in \Omega_{n}$ tal que $p .(z+X(t)) \not$ $p . I(t)$, pois basta tomar $z=\left(0, \ldots, 0, x_{i}, 0, \ldots, 0\right)$, isto é, podemos tomar um vetor $z$ tal que a $i$-ésima coordenada é não nula e estritamente positiva, e com todas as demais coordenadas nulas, vem daí que para $x_{i}$ suficientemente grande temos $p .(z+X(t)) \not p . I(t)$, o que contradiz o fato de $z+X(t) \in F(t)$, portanto temos que ter $p \geq 0$.

Afirmamos que para todo $t \in F(t), p . X(t) \gtrsim p . I(t)$.

Suponha que para algum $t \in T, p . X(t) \not{\star} p . I(t)$. Seja $\Phi=\left\{z \in{ }^{*} \Omega_{n}: p .(x+X(t))<\right.$ p.I(t)\}, temos $\Phi$ interno e $\mu(0) \subset \Phi$ e por esparramamento existe $z \in \Omega_{n} \subset{ }^{*} \Omega_{n}$ tal que $p .(z+X(t)) \nRightarrow z_{p} . I(t)$. Observe agora que por iv) e $\left.\gamma\right)$ temos $z+x(t) \succ \succ_{1} X(t)$ e portanto para todo $w \approx z+X(t), w \succ_{1} X(t)$.

Definindo $\Pi=\left\{(\delta \in R) ;\left(\forall w \in{ }^{*} \Omega_{n}{ }^{*} \rho(w, z+X(t))<\delta\right) w \succ_{t} X(t)\right\}$, temos $\Pi$ interno e $m(0) \subset \Pi$ por esparramamento existe $\delta>0(\delta \in R)$, e portanto existe $k \in N$ tal que $\frac{1}{k}<\delta,\left(\forall w \in{ }^{*} \Omega_{n}{ }^{*} \rho(w, z+X(t))<\frac{1}{k}<\delta\right) w \succ_{t} X(t)$ e portanto $z+X(t) \in F(t)$ o que nos leva a uma contradição. Logo para todo $t \in T, p . . Y(t) \gtrsim p . I(t)$.

Vamos mostrar agora que com excessão de no máximo para um conjunto negligenciável de consumidores $p . X(t) \approx p \cdot I(t)$.

Se para algum conjunto $S, S$ interno, tivermos $p . X(t) \not{\approx} p . I(t)$, então $\frac{1}{w} \sum_{t \in S} p . X(t) \gtrsim$ $\sum_{t \in S} p . I(t)$, pois, definindo $\phi_{t}=\left\{\varepsilon_{t} \in{ }^{*} R: p . X(t)>p . I(t)+\varepsilon_{l}\right\}$, temos $\phi_{t}$ interno $\mathrm{e}$ $m(0) \subset \phi_{t}$, pois para todo $t \in S$, tem-se $p . X(t)>p . I(t) \approx p . I(t)+\varepsilon_{l}$ por esparramamento existe $\varepsilon_{t}>0, \varepsilon_{t} \in R$, tal que $p . X(t)>p . I(t)+\varepsilon_{t}$. Sendo $S$ interno e finito, exite $0<\varepsilon_{t_{0}}=\min _{t \in S}\left\{\varepsilon_{t}, \varepsilon_{t} \in R\right\}$.

Vem daíque para todo $t \in S, p . X(t)>p . I(t)+\varepsilon_{t_{0}}, \log 0$

$$
\frac{1}{\omega} \sum_{t \in S} p . X(t)>\frac{1}{\omega} \sum_{t \in S} p . I(t)+\frac{|S|}{\omega} \varepsilon_{t_{0}}
$$

e, portanto,

$$
\frac{1}{\omega} \sum_{t \in S} p . X(t) \not{z} \frac{1}{\omega} \sum_{t \in S} p . I(t) .
$$


Mas, $\frac{1}{\omega} \sum_{t \in(T-S)} p \cdot X(t) \gtrsim \frac{1}{\omega} \sum_{t \in(T-S)} p . I(t)$, basta observar que tendo $p . X(t) \gtrsim p . I(t)$ para todo $t \in T$. Vem daí que

$$
\begin{aligned}
\frac{1}{\omega} \sum_{t \in T} p . X(t) & =\frac{1}{\omega} \sum_{t \in S} p \cdot X(t)+\frac{1}{\omega} \sum_{t \in(T-S)} p \cdot X(t) \not{z} \frac{1}{\omega} \sum_{t \in S} p . I(t)+\frac{1}{\omega} \sum_{t \in(T-S)} p . I(t) \\
& =\frac{1}{\omega} \sum_{t \in T} p . I(t),
\end{aligned}
$$

o que contradiz o fato de $X$ ser uma alocação.

Portanto $p . X(t) \approx p . I(t)$ com excessão de no máximo para um conjunto negligenciável de consumidores.

Para completar a prova vamos mostrar que $X(t)$ é maximal em $\beta_{p}(t)$ para todo $t$, isto é, $p . x \not z p . I(t)$ para $x \in F(t)$.

Pois se supormos p.x $\approx$ p.I(t) para $x \in F(t)$, temos $x \in \beta_{p}(t)$ e para todo $w \approx x$ temos $w \succ_{i} X(t)$. Definindo $\left.\left.\rho(w, x)<\delta\right) \cdot w \succ_{t} X(t), p . w<p . I(t)\right\}$, temos $\Delta$ interno, e por esparramamento existe $\delta>0, \delta \in R$, e portanto existe $k \in N$, tal que $\frac{1}{k}<\delta$ e $\left.\left(\forall w \in{ }^{*} \Omega_{n}{ }^{*} \rho(w, x)<\frac{1}{k}<\delta\right) w \succ_{t} X(t)\right)$ e portanto $x \succ_{t} X(t)$ não maximal em $\beta_{p}(t)$.

Afirmamos que $p \not 2$

Suponha que não, isto é, suponha sem perdas de generalidades, que $p_{1} \approx 0$. Observe agora que o vetor $p$ é "standard" não nulo, e portanto alguma coordenada de $p$ é não infinitesimal, sem perda de generalidade, seja $p_{2} \gtrsim 0$.

Mas $\frac{1}{\omega} \sum_{t \in T} I^{2}(t) \not{\approx} 0$, onde $I^{2}(t)$ segunda coordenada de $I(t)$; visto que $\frac{1}{\omega} \sum_{t \in T} I(t) \not{\not}$ 0 . Sendo $X$ uma alocação segue que $\frac{1}{\omega} \sum_{t \in T} X(t) \approx \frac{1}{\omega} \sum_{t \in T} I(t)$, vem daí que $\frac{1}{\omega} \sum_{t \in T} X^{2}(t) \gtrsim$ 0 e portanto existe um conjunto não negligenciável de consumidores $S, S$ interno, para o qual $X^{2}(t) \not{\approx} 0$, onde $X^{2}(t)$ segunda coordenada de $X(t)$ com $t \in S$.

Agora, para todo consumidor $t$, segue de iv) e $\gamma)$ que $X(t)+(1,0, \ldots, 0) \succ_{t} X(t)$. Escolhendo arbitrariamente $t \in S$, podemos obter $\varepsilon>0, \varepsilon \in R$, suficientemente pequeno para termos $X(t)+(1,-\varepsilon, 0, \ldots, 0) \succ_{t} X(t)$. Pois, basta definir $\varphi=\{\varepsilon \in$ $\left.{ }^{*} R: X(t)+(1,-\varepsilon, 0, \ldots, 0) \succ_{t} X(t)\right\}$, temos $\varphi$ interno, $m(0) \subset \varphi$ pois para todo 
$w=X(t)+(1,-\varepsilon, 0, \ldots, 0)$, onde $\varepsilon \in m(0)$ temos $w \approx X(t)+(1,0, \ldots, 0)$ e portanto $w \succ_{1} X(t)$ (observe que para todo $\varepsilon \in m(0), X(t)+(1,-\varepsilon, 0, \ldots 0) \in{ }^{*} \Omega_{n}$, já que $\left.X^{2}(t) \not 0\right)$, temos então por esparramamento que existe $\varepsilon>0, \varepsilon \in R$ tal que $\mid X(t)+(1,-\varepsilon, 0, \ldots, 0) \succ_{t} X(t), \log 0, X(t)+(1,-\varepsilon, 0, \ldots, 0) \in F(t)$ e temos

$$
p . I(t) \lesssim p \cdot[X(t)=(1,-\varepsilon, 0, \ldots, 0)]=p \cdot X(t)+p^{1}-p^{2} \varepsilon \not{\not} p . . Y(t),
$$

isto é, $p . I(t) \not{\not} p . X(t)$, para todo $t \in S$.

Sendo $\frac{|S|}{\omega} \not 0$, isto contradiz o fato de $p . X(t) \approx p . I(t)$, exceto para um conjunto não negligenciável de consumidores. Logo $p \not \approx 0$.

Agora suponha $x \in F(t)$ e $I(t) \not{\not} 0$; então $p . I(t) \not \approx 0$, pois $p \not \approx 0$. Visto que $p . x \not{\not}$ p.I(t), segue que p.x $\ddot{\approx} 0$ e portanto existe $j$ tal que $x_{j} \not{\approx} 0$, sem perda de generalidade, seja $j=1$. Então $x-(\varepsilon, 0, \ldots, 0) \in F(t)$ para $\varepsilon$ suficientemente pequeno. Pois, definindo $\eta=\left\{\varepsilon \in{ }^{*} R: x-(\varepsilon, 0, \ldots, 0) \succ_{1} X(t), x-(\varepsilon, 0, \ldots, 0) \in{ }^{*} \Omega_{n}\right\}$, temos $\eta$ interno $\mathrm{e}$ $m(0) \subset \eta$, pois para todo $\varepsilon \in m(0)$, temos $x-(\varepsilon, 0, \ldots, 0) \approx x, x-(\varepsilon, 0, \ldots, 0) \in{ }^{*} \Omega_{n}$ e portanto $x-(\varepsilon, 0, l ;$ dots $s, 0) \succ_{t} X(t)$ e por esparramamento existe $\varepsilon>0, \varepsilon \in R$ tal que $x-(\varepsilon, 0, \ldots, 0) \in F(t)$. Então, $p . I(t) \lesssim p \cdot[x-(\varepsilon, 0, \ldots, 0)]=p . x-p^{1} . \varepsilon \approx \varpi^{2} p . x$, isto é, $p . I(t) \not p . x$. Se $I(t) \approx 0$, é claro que $p . x \not z p . I(t)$.

Finalmente, suponha $I(t) \approx 0$ e $x \approx 0$. Dado que $x \in F(t)$, temos $I(t) \in F(t)$.

Se o conjunto $S$ de consumidores t para os quais $I(t) \in F(t), S$ for negligenciável, então pode ser ignorado. Se por outro lado, $S$ for não negligenciável, então $I(t)$ domina $X(t)$ via coalizão $S$, contradizendo o fato de $X$ estar no "core". O que conclui a demonstração.

\subsection{Réplicas de uma economia}

(uma abordagem não "standard")

Nesta seção abordaremos réplicas econômicas (vide capítulo 1, definição 1.18), enunciaremos o teorema de Brown and Robinson que relaciona uma alocação Walrasiana para uma 
economia de trocas $\varepsilon$, $\operatorname{com} m$ "agentes", com o "core" de suas réplicas.

Definição 2.13 Seja $H=\left\{\varepsilon_{i}\right\}_{i \in I}$ uma família não limitada de economias de trocas com um número "standard" de agentes econômicos, isto é, para cada $k \in N$, existe $i \in I$ tal que $|\varepsilon|$, o número de "traders" da i-ésima economia, é maior que k.

Suponha que $H$ satisfaça as seguintes propriedades:

a) A dotação inicial, $e_{i}, t=1, \ldots, i$, dos "agentes" de $\varepsilon_{i} \in H, i \in I$, é uniformemente limitada superiomente, isto é, existe $n \in R$ tal que $I(t) \leq n$, para todo $t=1 \ldots, i$, para toda economia $\varepsilon_{i} \in H$.

b) A dotação inicial $e_{t}, t=1, \ldots, i$, do "agentes" de $\varepsilon_{i} \in H, i \in I$, é uniformente limitada inferiormente acima do zero, isto é, existe $m \in R, 0<m<n$, tal que $e_{t}>m$, para todo $t=1, \ldots, i$, para toda economia $\varepsilon_{i} \in H$.

c) A relação de preferência de cada "agente" $t$ em $H$ é

c') Irreflexiva (vide apêndice A);

c") Contínua, isto é, para todo $x, y \in \Omega$, os conjuntos $\left\{z \in \Omega_{n}: z \succ_{1} x\right\},\left\{z \in \Omega_{n}\right.$ : $\left.y \succ_{1} z\right\}$ são aberto em $R^{n}$

c"') Fotemente monótona, isto é, se $x \gg y \Rightarrow x \succ_{t} y$.

d) A família das relações de preferências de todos os "agentes" em $H$ é equimonotônica sobre $\Omega_{n}$, isto é, para todo $x \geq 0$ e para todo $y>0$ existe $\delta>0$ e para todo $i \in I$ e para todo $t \in \varepsilon_{i}$ temos que se $z \in B(x+y, \delta)$ e $w \in B(x, \delta) \Rightarrow z \succ_{t} w$.

Definição 2.14 Dadas uma economia de trocas $\varepsilon=\left\{I(t), \succ_{t} ; t=1, \ldots, m\right\}$ "standard" ou nào "standard", uma troca $X(t)$ e um vetor de preços $p \in{ }^{*} \Omega_{n}$. (Obs.: chamaremos de economia de trocas "standard" aquelas cujo número de "agentes" é um natural 
"standard"). Definimos os seguintes conjuntos para cada real "standard" positivo $\delta$ :

$$
\begin{aligned}
& D_{\delta}^{p}(X)=\{t \in \varepsilon: p X(t)-p I(t) \geq \delta\} \\
& F_{\delta}^{p}(X)=\left\{t \in \varepsilon:\left(\exists y \in \Omega_{n}\right) p y \leq p I(t) \wedge\left(\forall w \in B(y, \delta) w \succ_{t} X(t)\right\}\right. \\
& G_{\delta}^{p}(X)=D_{\delta}^{p} \cup F_{\delta}^{p} .
\end{aligned}
$$

Podemos interpretar os conjuntos definidos acima como segue:

$D_{\delta}^{p}(X)$ é o conjunto dos "agentes" cuja compra da cesta $X(t)$ ao preço $p$ tem seu budget set violado por um valor $\delta$;

$F_{\delta}^{p}(X)$ é o conjunto dos "agentes" em $\varepsilon$ que podem adquirir uma "commodity" $y_{1}$, ao vetor de preços $p$, cujas quantidades são finitias, sem violar o budget set dado por $p$, e tal que toda "commodity" $z \in B\left(y_{t}, \delta\right)$ é preferível a $X(t)$.

Teorema 2.2 Seja $K=\left\{\varepsilon_{i}, X_{i}\right\}_{i \in I}$ e $H=\left\{\varepsilon_{i}\right\}_{i \in I}$. Suponha $H$ uma família não limitada de economias de trocas satisfazendo as propriedades acima. Sejam $X_{i}$ uma família de alocaçôes, onde $X_{i} \in \ell\left(\varepsilon_{i}\right)$, e sejam $X_{i}$ uniformemente limitada por cima. Então, para todo $\delta>0$, existe $n \in N$ tal que para toda economia $\varepsilon_{i} \in H$, se $\left|\varepsilon_{i}\right|>n$ existe um vetor de preços p tal que $\frac{\left|G_{\delta}^{p}\right|}{\left|\varepsilon_{i}\right|}<\delta$.

Antes de provarmos o teorema 2.2 acima, faremos algumas observações e daremos mais algumas definições.

As noções de "core" definidas para economias de trocas "standard" e não "standard" diferem na definição da alocação e bloqueios (vide definição 1.11, capítulo 1, e definição 2.8, capítulo 2). Mas o conceito de "core" para economias de trocas "standard" é também significativo nas economias de trocas não "standard", o que será demonstrado no que segue.

"Q-core" 
Definição 2.15 Chamaremos de "Q-core" (leremos quase "standard core") o conceito de "core standard" quando transferido para economias de trocas nào "standard".

\section{"Q-Blocks"}

Definição 2.16 Seja $\varepsilon=\left\{I(t), \succ_{t}, t=1, \ldots, \omega\right\}$ economia de trocas não "standard" satisfazendo as propriedades listadas da definiçâo 2.1, capítulo 2. Seja $\phi=\left\{Y \in{ }^{*} \Omega_{n}^{T}: Y\right.$ é uma troca e $\left.\sum_{t \in T} Y(t) \leq \sum_{t \in T} I(t)\right\}$. Se $Z, Y \in \phi$, diremos que $Z$ "Q-blocks" $Y$ via coalizão $S \subseteq T$ se, e somente se, $\sum_{t \in S} Z(t)=\sum_{t \in S} I(t)$ e para todo $t \in S, Z(t) \succ_{t} Y(t)$. Se $Y \in \phi$ e $Y$ é não "Q-blocked" por qualquer $Z \in \phi$, diremos que $Y$ está no "Q-core".

Provaremos o teorema 2.2 acima, provando, primeiramente, o lema 2.3 que enunciaremos a seguir que afirma que trocas "standard" limitadas no "Q-core" de $\varepsilon$ estão no "core" de $\varepsilon$.

Lema 2.3 Se existe $\delta \ngtr 0$ tal que para todo $t \in T$ e para todo $I^{j}(t) \geq 0$. entáa toda troca "standard" limitada no "Q-core" de $\varepsilon$ está contida no "core" de $\varepsilon$.

Prova: Seja $X$ uma troca "standard" limitada no "Q-core" de $\varepsilon$ e não no "core" de $\varepsilon$. Então existe uma coalizão $S, S$ interno, e uma alocação $Y$ tal que para todo $t \in S$, $Y(t) \succ \succ_{t} X(t) \mathrm{e}$

$$
\frac{1}{\omega} \sum_{t \in S} Y(t) \approx \frac{1}{\omega} \sum_{t \in S} I(t) \quad \operatorname{com} \frac{|S|}{\omega} \not \approx 0 .
$$

De fato, de $\frac{1}{\omega} \sum_{t \in S} Y(t) \approx \frac{1}{\omega} \sum_{t \in S} I(t)$, temos:
(a) $\frac{1}{\omega} \sum_{t \in S} Y(t) \leq \frac{1}{\omega} \sum_{t \in S} I(t) \quad$ ou
(b) $\frac{1}{\omega} \sum_{t \in S} Y(t)>\frac{1}{\omega} \sum_{t \in S} I(t)$.

Caso (a) 
Se $\frac{1}{\omega} \sum_{t \in S} Y(t) \leq \frac{1}{\omega} \sum_{t \in S} I(t)$, então $\sum_{t \in S} Y(t) \leq \sum_{t \in S} I(t)$, pois basta multiplicar ambos os lados da desigualdade por $\omega$. Temos então que a troca

$$
Z(t)= \begin{cases}Y(t) & , t \in S \\ I(t) & , t \notin S\end{cases}
$$

é uma alocação com ${ }^{\circ}\left(\frac{1}{\omega} \sum_{t \in S} Z(t)\right) \ldots \circ\left(\frac{1}{\omega} \sum_{t \in S} I(t)\right)$ e $Z(t) \succ_{t} X(t)$ para $t$ em $S$, e portanto $Z$ "Q-blocks" $X$, o que contradiz o fato de $X$ estar no "Q-core" de $\varepsilon$.

Caso (b)

Se supormos que para algum $j$ que $\frac{1}{\omega} \sum_{t \in S} Y^{j}(t)>\frac{1}{\omega} \sum_{t \in S} I^{j}(t)$ e tendo por hipótese que existe $\delta>0$ tal que para todo $j, I^{j}(t) \geq \delta$, temos que $\frac{1}{\omega} \sum_{t \in S} I^{j}(t) \geq \frac{1}{\omega} \sum_{t \in S} \delta=$ $\frac{|S|}{\omega} \delta>0$ e portanto $\frac{1}{\omega} \sum_{t \in S} Y^{j}(t)>0$.

Defina para todo $n \in N$ o conjunto $B_{n}=\left\{t \in S: Y^{j}(t) \geq \frac{1}{n}\right\}$, temos que para todo $n \in N$ que $B_{n}$ interno e $B_{n} \subset B_{n+1}$.

Suponha que para todo $n \in N, \frac{\left|B_{n}\right|}{\omega} \approx 0$. Ent.ão existe $v \in{ }^{*} N \backslash N$ tal que $\frac{\left|B_{\nu}\right|}{\omega} \approx 0$, pois definindo $\Gamma=\left\{k \in{ }^{*} N:\left(\forall j \in{ }^{*} N\right),(k:<j) \Rightarrow B_{k} \subset B_{j}, 0 \leq \frac{\left|B_{k}\right|}{\omega} \leq \frac{1}{k}\right\}$, temos $\Gamma$ interno (vide teorema de Kiesler, apêndice C), $N \subseteq \Gamma$, c por esparramamento (vide apêndice C), existe $\nu \in{ }^{*} N \backslash N$ tal que $0 \leq \frac{\left|B_{\nu}\right|}{\omega} \leq \frac{1}{\nu} \Rightarrow \frac{\left|B_{\nu}\right|}{\omega} \approx 0$. Vem daí que:

$$
\frac{1}{\omega} \sum_{t \in S} Y^{j}(t)=\frac{1}{\omega} \sum_{t \in B_{\nu}} Y^{j}(t)+\frac{1}{\omega} \sum_{t \in S \backslash B_{\nu}} Y^{j}(t) \approx \frac{1}{\omega} \sum_{t \in S \backslash B_{\nu}} Y^{j}(t) \leq \frac{|S|}{\omega} \frac{1}{\nu} \approx 0,
$$

isto porque se temos $t \in S \backslash B_{\nu} \Rightarrow Y^{j}(t)<\frac{1}{\nu}$, pois $Y$ é "standard" limitada. O que contradiz o fato de $\frac{1}{\omega} \sum_{t \in S} Y^{j}(t)>0$. Conseqüentemente, existe $n \in N$ tal que $\frac{\left|B_{n}\right|}{\omega}>0$.

Seja $\alpha=\left|B_{n}\right|, \gamma=\frac{1}{\omega} \sum_{t \in S} Y^{j}(t)-\frac{1}{\omega} \sum_{t \in S} I^{j}(t)$; e para todo $t \in B_{n}, \delta_{t}=\gamma \frac{\omega}{\alpha}$. Observe que pelas considerações feitas no início do caso b) $\gamma$ é infinitesimal. Defina

$$
Z^{j}(t)= \begin{cases}Y^{j}(t)-\delta & , t \in B_{n} \\ Y^{j}(t) & , t \in S \backslash B_{n}\end{cases}
$$

Para $j \neq i, j, i \in S$, seja $Z^{\prime}(t)=Y^{i}(t)$, para todo $t \in S$; finalmente seja $Z(t)=I(t)$ para todo $t \in T \backslash S$. 
Agora, se $\frac{\left|B_{n}\right|}{\omega}>0 \Rightarrow \frac{\omega}{\alpha}$ é finito e portanto $\delta_{t}=\frac{\omega}{\alpha} \gamma$ é infinitesimal. Temos então que $t \in S, Z(t) \approx Y(t)$ e pela propriedade $\gamma^{\prime}$ presente na definição 2.2 , no início do capítulo 2 , temos que $Z(t) \succ_{t} X(t), \forall t \in S$, pois $Z(t) \gg_{t} X(t)$, isto é, para todo $y \in \mu(Z) \Rightarrow y \succ_{t} X(t)$. Mas $Z \in \phi$, pois

$$
\frac{1}{\omega} \sum_{t \in S} Z^{j}(t)=\frac{1}{\omega} \sum_{t \in B_{n}} Z^{j}(t)+\frac{1}{\omega} \sum_{t \in S \backslash B_{\nu}} Z^{j}(t)=\frac{1}{\omega} \sum_{t \in S} Y^{j}(t)-\gamma=\frac{1}{\omega} \sum_{t \in S} I^{j}(t) .
$$

(Obs.: se existir mais de um $j$ para o qual $\frac{1}{\omega} \sum_{t \in S} Y^{j}(t)>\frac{1}{\omega} \sum_{t \in S} I^{j}(t)$, repita a construção), o que contradiz o fato de $X(t)$ estar no "Q-core" e portanto para toda troca limitada $X$ no "Q-core" $\varepsilon, X$ está no "core" de $\varepsilon$.

A prova do teorema 2.2 segue imediatamente.

Suponha que o teorema é falso, então, para algum $\delta>0$ e para todo $m \in N$, existe $\left(\varepsilon_{i}, X_{i}\right) \in K$, tal que $\left|\varepsilon_{i}\right|>m, \operatorname{com} X_{i}$ tendo a propriedade de para todo vetor de preços $p, \frac{\left|G_{\delta}^{p}(X)\right|}{\left|\varepsilon_{i}\right|} \geq \delta$. Aplicando o princípio de transferência temos que para algum $\delta \in R, \delta>0$, e para todo $m \in{ }^{*} N$, existe $\left(\varepsilon^{\prime \prime}, X\right) \in{ }^{*} K$, tal que $\left|\varepsilon^{\prime \prime}\right|>m$, e $X$ está no "core" de $\varepsilon^{\prime \prime}$ se $\varepsilon^{\prime \prime}$ for uma economia "standard", e $X$ troca "standard" limitada no "Q-core" se $\varepsilon$ ", se $\varepsilon$ " for economia não "standard". Em qualquer caso, $X$ tem a propriedade de que para todo vetor de preços $p, \frac{\left|G_{\delta}^{p}(X)\right|}{\left|\varepsilon^{\prime \prime}\right|} \geq \delta$.

Escolhendo $m$ como um $\omega \in{ }^{*} N \backslash N$, existe $\left(\varepsilon^{\prime \prime}, X\right) \in{ }^{*} K$ satisfazendo as propriedades acima. $\varepsilon^{\prime \prime}$ economias de trocas não "standard" e $X$ não é um equilíbrio competitivo para $\varepsilon^{\prime \prime}$, pois para todo $t \in G_{\delta}^{p}(X)$, temos que existe $Y \in \Omega_{n}$ tal que $p Y(t) \leq p I(t)$ e para todo $w \in S(Y, \delta)$, temos $w \succ_{t} X(t)$, e pelo teorema $2.1, X \notin \ell(\varepsilon)$, o que contradiz o lema 2.3, pois tomamos $X$ no "Q-core" da economia $\varepsilon$ ".

O teorema 2.2 afirma que alocações no "core" de uma economia não "standard" são também alocações walrasianas ou competitivas que satisfazem a totalidade dos agentes da economia.

A seguir enunciaremos e demonstraremos o teorema de Brown and Robinson, que 
apresenta uma alternativa de prova para o teorema limite de Debreu-Scarf.

Teorema 2.3 (Brown and Robinson) Seja $\varepsilon$ uma economia de trocas. Se todo "trader" tem suas preferências representadas por uma funçấo utilidade com as propriedades de forte monotonicidade, irreflexividade, e continua, e todos os "trader" saio possuidores de dotaçôes iniciais estritamente positivas, entâo uma alocaçào X é uma alocaçào competitiva para $\varepsilon$, ou seja, $X$ é um equilíbrio walrasiano para $\varepsilon$ se. e somente se, $X^{(r)}$ está no "core" de $\varepsilon^{(r)}$ para todo $r \geq 1$.

Prova: Seja $X^{(r)}$ uma alocação no "core" de $\varepsilon^{(r)}$ para todo $r \geq 1$, temos então que existe $n \in \Omega_{n}$ tal que $X^{(r)}(t) \leq n$, para todo $t \in\left|\varepsilon^{(r)}\right|$. Aplicando o princípio de transferência, t.emos que existe $X^{(\sigma)}$ troca "standard" limitada no "Q-core" de $\varepsilon^{(\sigma)}$ para todo $\sigma \in{ }^{*} N \backslash N$.

Seja $\omega \in{ }^{*} N \backslash N$, temos que $\varepsilon^{(\omega)}$ satisfaz todas as hipóteses e resultados do teorema 2.1 e do lema que precede a demonstração do teorema 2.2. Ist,o é, existe um vetor de preços $p$ tal que $\left(p, X^{(\omega)}\right)$ é um equilíbrio competitivo para $\varepsilon^{(\omega)}$ (Obs.: o teorema 2.1, capítulo 2 , garante que o vetor de preços $p \gg 0$ e "standard").

Afirmamos que $\left(p, X^{(1)}\right)$ é um equilíbrio competitivo para $\varepsilon^{(1)}$. Se não, pelo menos um "trader" $t$ tem uma cesta de bens $y$ em seu "budget set" tal que $y \succ_{t} X(t)$.

Vem daí que na $\omega$-ésima réplica existem pelo menos $m \omega$ "trader" que preferem y a $X^{(\omega)}$, e sendo a preferência dos $m \omega$ contínua, existe $\delta>0$ tal que para todo $w \in S(y, \delta)$ $w$ é preferido a $X^{(\omega)}$, o que $w$ é preferido a $X^{(\omega)}$, o que contradiz o fato de $\left(p, X^{(\omega)}\right)$ ser um equilíbrio competitivo para $\varepsilon^{(\omega)}$. O que prova a afirmação. 


\title{
Apêndice A
}

\section{"Commodities" e relações de preferências}

\author{
"COMMODITY"
}

Definição A.1 Chamaremos de "commodity" a todo bem ou serviço ao qual especificamos suas características físicas, local e data de sua produção. Isto nos permitirá distinguir os bens não só por suas características físicas, mas também pela data e localização em que estarão disponíveis.

Exemplo 1: 1 ton. de soja produzida no Brasil será considerado distinta de 1 ton. de soja produzida nos E.U.A. mesmo que estejam disponíveis em uma mesma data. Da mesma forma consideramos 1 ton. de soja produzida em regiões distintas de um pais "commodities" distintas mesmo estando disponíveis em uma mesma data e em uma mesma safra..

Sendo uma "commodity" um bem, podemos expressar suas quantidades por um número. Assumiremos que tal quantidade pode ser infinitamente divisível e assim representaremos as quantidades de uma "commodity" por números reais. Assumiremos que o número de "commodities" distintas disponíveis em uma economia é finito.Fazendo isto estaremos mais próximos das condições reais de mercado, bem com garantiremos a possibilidade 
técnica de exprimir tais "commodities" por um elemento em $R_{+}^{n}$, onde $n$ é o número de "commodities" disponíveis, o elemento $x=\left(x_{1}, \ldots, x_{n}\right) \in R_{+}^{n}$ será chamado de cesta de "commodities" com cada coordenada representando a quantidade de um determindado bem. O conjunto $R_{+}^{n}$ será denominado espaço das "commodities".

\section{CONJUNTO CONSUMO E PREFERENCIAS}

Definição A.2 Um conjunto de consumo $X$ é um subconjunto não vazio do espaço das "commodities", fechado, convexo.

Seja $A \subset N$ um conjunto com $m$ elementos, um elemento $a \in A$ será chamado de consumidor ou "trader", e a cada $a \in A$ associaremos um conjunto de consumo $X_{a} \subset R^{n}$, que chamaremos de conjunto de consumo do consumidor a. Queremos dizer com isto que, ignorando algumas restrições, o consumidor pode consumir qualquer cesta de bens pertencente a $X_{a}$.

Definição A.3 Uma relação de preferência é definida por um par $(X, \succ)$, onde $X$ é um conjunto de consumo e $\succ$ é uma relação binária em $X$, tal que $\succ$ é um aberto em $X \times X$ e para todo $(x, y) \in \succ$ diremos que a cesta $x$ é preferivel à cesta $y$, ou simplesmente que $x$ é preferivel a $y$, para representar tal fato usaremos a notação mais sugestiva $x \succ y$. Introduziremos também a relação $\approx$. Assim, $x \approx y$ significa que o agente é indiferente entre as cestas $x$ e y. Combinaremos $\succ e \approx$ na relação $\succeq$ que significará preferível ou indiferente. Exigiremos que $\succeq$ seja um conjunto fechado de $X \times X$.

Exigiremos as seguintes propriedade para $\succ$ :

a) Irreflexividade: para todo par $(x, x)$ tem-se $(x, x) \notin \succ$, e escreveremos $x \nsucc x \mathrm{e}$ leremos $x$ não é preferível a $x$. 
b) Transitividade: para todo $x, y, z \in X$, se $x \succ y, y \succ z \Rightarrow x \succ z$. Seja $\chi$ o conjunto de todos os conjuntos de consumo, o conjunto de todas as relações de preferencia em $X \in \chi$ com as propriedades acima será denotado por $\mathcal{P}$.

Observação Ao exigirmos que $\succ$ seja transitiva e irreflexiva, estamos garantindo que $\succ$ é uma ordem parcial fraca em $X$, isto é ,

\section{EXEMPLO 2}

Defina $\succ$ relação binária em $X=R_{++}^{2}=\left\{x \in R^{2} \mid x_{i}>0, i=1,2\right\}$, por

$$
x=\left(x_{1}, x_{2}\right) \succ y=\left(y_{1}, y_{2}\right) \Leftrightarrow x_{1}>y_{1} \text { ou } x_{1}=y_{1} \quad \text { e } \quad x_{2}>y_{2} .
$$

a) $\succ$ é transitiva: para todo $x, y, z \in X$ tem-se que se $x \succ y$ e $y \succ z \Rightarrow x \succ z$, pois

Caso 1) se $x_{1}>y_{1}, y_{1}>z_{1} \Rightarrow x_{1}>z_{1}$ (pela transitividade da relação de ordem usual em $R$ ) e daí $x \succ z$;

Caso 2) se $x_{1}>y_{1}, y_{1}=z_{1} \Rightarrow x_{1}>z_{1}$ e daí $x \succ z$;

Caso 3) se $x_{1}=y_{1}, x_{2}>y_{2}$ e $y_{1}>z_{1} \Rightarrow x_{1}>z_{1}$ e daí $x \succ z$;

Caso 4) se $x_{1}=y_{1}, x_{2}>y_{2}$ e $y_{1}=z_{1}, y_{2}>z_{2} \Rightarrow x_{2}>z_{2}$ e daí $x \succ z$.

b) $\succ$ é irreflexiva

Prova: Imediato.

c) $\succ$ é completa: pois para todo $x, y$ em $X$ com $x \neq y$, tem-se $x \succ y$ ou $y \succ x$.

Prova: Imediata.

Temos assim que $\succ$ é completa e $\succ$ é um aberto em $X \times X$ e, portanto, $\succ$ é uma preferência em $X$. 
A relação binária acima é chamada de ordem lexicográfica. Para toda relação de preferência $(X, \succ)$ em $\mathcal{P}$, associaremos o conjunto

$$
F:=\left\{(x, y) \in R^{n} \times R^{n} \mid x, y \in X, x \nsucc y\right\} .
$$

Propriedades.

a) $F$ é um conjunto fechado em $R^{n} \times R^{n}$.

Prova: Basta observar que que $F$ é o complementar de $\succ$ que é um aberto em $X \times X$.

b) O conjunto $\left\{x \in R^{n} \mid \exists y,(x, y) \in F\right\} \in \chi$, conjunto de todos os conjuntos de consumo.

Prova: imediata.

\section{ALGUMAS PROPRIEDADES}

Uma relação de preferência pode ser:

a) Localmente não saciada.

Uma relação de preferência $(X, \succ)$ é dita localmente não saciada se para todo $x \in X$ e toda vizinhança $U$ de $x$ existe $y \in X \cap U$ tal que $y \succ x$.

\section{EXEMPLO 3}

Seja $X=R^{2}$, seja $(X, \succ)$ relação de preferência dada pela ordem lexicográfica dada no exemplo 1. Temos que $X$ é um aberto em $R^{2}$, $\log$ para todo $x \in X$, existe $\delta>0$ tal que $B_{\delta}(x) \subset X$. Seja $y=\left(y_{1}, y_{2}\right)$, tal que $y_{1}>x_{1}, y_{1}-x_{1}<\delta$ e $y_{2}=x_{2}$, temos então que $y \succ x$ e $\|x-y\|=\sqrt{\left(x^{1}-y_{1}\right)^{2}+\left(x_{2}-y_{2}\right)^{2}}<\sqrt{\delta^{2}}=\delta$ e portanto $\succ$ localmente não saciada.

Denotaremos o conjunto de todas as relações de preferências localmente não saciada por $\mathcal{P}_{\text {Ins }}$. Observe que $\mathcal{P}_{\text {ins }} \subset \mathcal{P}$.

b) Monótona. 
Uma relação de preferência $(X, \succ)$ é dita monótona ou monotônica se para todo $x, y \in$ $X$, com $x \neq y$, tem-se que se $x<y \Rightarrow y \succ x$.

Denotaremos o conjunto de todas as relções de preferências monótonas por $\mathcal{P}_{m o}$. Observe que $\mathcal{P}_{\text {mo }} \subset \mathcal{P}$.

Para uma relação de preferência em $\mathcal{P}$, diremos que $x$ é indiferente a $y$, caso $x \neq y$ e $y \nsucc x$ denotaremos tal fato por $x \approx y$. O par $(X, \approx)$ determina uma relação de indiferença com as seguintes propriedades:

a) Reflexiva: $x \approx x, \forall x \in X$;

b) Transitiva: se $x \approx y$ e $y \approx z \Rightarrow x \approx z$;

c) Simétrica: se $x \approx y \Rightarrow y \approx x$.

Dados $x, y \in X$, se temos $y \nsucc x$, ter.nos então duas possibilidades a considerar: $x \succ y$ ou $x \approx y$ representaremos tal fato com a seguinte notação $x \succeq y$, onde lê-se que $x$ é preferível ou indiferente a $y$.

O par $(X, \succeq)$ determina uma relação binária com as seguintes propriedades:

a) Reflexiva: $x \succeq x, \forall x \in X$;

b) Transitiva: $x \succeq y, y \succeq z \Rightarrow x \succeq z$;

c) Completa: $\forall x, y \in X$, tem-se $x \succeq y$ ou $y \succeq x$ (queremos dizer com isto que para quaisquer duas cestas tomadas no conjunto de consumo, estas podem ser comparadas).

Para a relação de preferência $\succeq$ podemos ter ainda as seguintes propriedades:

a) Convexidade: $\forall z \in X$, o conjunt. $\{x \in X \mid x \succeq y\}$ é convexo.

b) Fortemente convexa: $\forall x, y \in X, \operatorname{com} x \approx y, x \neq y, \forall \lambda \in[0,1]$, tem-se $\lambda x+(1-\lambda) y \succ$ $x$. 
O conjunto de todas as preferências convexas (fortemente convexas) em $\mathcal{P}$ será denotado por $\mathcal{P}_{c o}\left(\mathcal{P}_{s c o}\right)$

\section{FUNCCÕES UTILIDADE}

Quando possível procuraremos a representação de uma relação de preferência por uma função, tais funções são denominadas funções utilidade.

Definição A.4 Seja $(X, \succ)$ uma relação de preferência. Uma função utilidade para $(X, \succ)$ é uma função contínua $u: X \rightarrow R$, tal que $x \succ y \Leftrightarrow u(x)>u(y)$.

É imediato que dada uma função contínua $u: X \rightarrow R$ podemos trivialmente relacionar uma relação de preferência $\succ_{u}$ a $u$ por $x \succ_{u} y \Leftrightarrow u(x)>u(y)$.

É importante notar que nem toda relação de preferência em $X \subset R^{n}$ pode ser representada por uma função utilidade; por exemplo: seja $\left(R_{+}^{2}, \succ\right)$ relação de preferência dada pela ordem lexicográfica (vide exemplo1, preferências). Suponha que exista $u: R_{+}^{2} \rightarrow R$ representando $\succ$. Sejam $x=(r, 1)$ e $y=(r, 0) \in R_{+}^{2}$, temos $x \succ y$, pois da definição de $\succ$ o fato de $r=r, 1>0$ garante a preferência de $x$ a $y$. Segue que sendo $u$ função utilidade para $\succ$, devemos ter $u(x)>u(y)$. Sendo $Q$ conjunto dos racionais denso em $R$, existe racional, $q_{r}$, tal que $u(x)>q_{r}>u(y)$.

Defina $\varphi: R \rightarrow Q$ por $\varphi(r)=q_{r}$. Seja $r^{\prime}>r, r^{\prime}$ real; analogamente temos $x^{\prime}=$ $\left(r^{\prime}, 1\right) \succ y^{\prime}=\left(r^{\prime}, 0\right)$ e assim, existe $\varphi\left(r^{\prime}\right)=q_{r}$, tal que $u\left(x^{\prime}\right)>\varphi\left(r^{\prime}\right)>u\left(y^{\prime}\right)$. Segue daí que sendo $y^{\prime} \succ x$, pois $r^{\prime}>r$; teremos que $u\left(x^{\prime}\right)>\varphi\left(r^{\prime}\right)>u\left(y^{\prime}\right)>u(x)>\varphi(r)>u(y) \Rightarrow$ $\varphi\left(r^{\prime}\right)>\varphi(r) \Rightarrow \varphi$ crescente e portanto $\varphi$ injetora, podemos concluir então que o conjunto dos números reais é enumerável, o que nos leva a um absurdo.

Daremos a seguir as condições necessárias e suficientes para que exista uma função utilidade que represente uma preferência.

Definição A.5 Uma relação binária $S$ sobre um conjunto $X$ é: 
a) Uma ordem fraca se, e somente se, $S$ sobre $X$ é

a') Assimétrica: se $(x, y) \in S \Rightarrow(y, x) \notin S, \forall x, y \in X$;

a") Transitiva negativa: se $(x, y) \notin S e(y, z) \notin S \Rightarrow(x, z) \notin S, \forall x, y, z \in X$.

b) Uma ordem estrita se,e somente se, $S$ sobre $X$ é:

b') Uma ordem fraca;

b") Fracamente conexa: se $x \neq y \Rightarrow(x, y) \in S$ ou $(y, x) \in S$, isto é, todos os elementos de $X$ sâo comparáveis segundo a ordem imposta pela relação binária $S$.

c) Uma equivalência se, e somente s屯, $S$ sobre $X$ é:

c) Reflexiva: $(x, x) \in S, \forall x \in X$;

c") Simétrica: $(x, y) \in S$;

c") Transitiva: se $(x, y) \in S e(y, z) \in S \Rightarrow(x, z) \in S, \forall x, y, z \in X$.

Teorema A.1 Seja $\succ$ uma ordem fraca em $X$.

a) para todo $x, y \in X$ uma e somente uma das condições abaixo é satisfeita.

a') $x \succ y$;

a") $y \succ x$;

a"') $x \approx y($ lê-se $x$ é indiferente a $y)$.

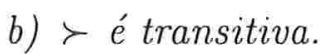

c) ₹ é uma relaçâo de equivalência em $X$.

d) $y \succ x, y \approx z \Rightarrow z \succ x e x \approx y, z \succ y \Rightarrow z \succ x$.

e) $\succ e ́$ transitiva e conexa (conexa: $x \succ y$ ou $y \succ x$ sendo ambas possiveis - para todo $x, y \in X)$. 
f) A relaçấo $\succeq^{\prime}$ sobre $X / \approx$ (conjunto das classes de equivalência de $X$ sob $\approx$ ) definida por $b \succ^{\prime} a \Leftrightarrow y \succ x$ para algum $x \in a$ e $y \in b$; é uma ordem estrita.

Prova: Vide Utility Theory for Decision Making, Peter C. Fishburn, pag. 13.

Teorema A.2 Seja $\succ$ uma ordem fraca em $X$ e X/ $\approx$ contável, então exisce uma função utilidade $u: X \rightarrow R$ tal que $y \succ x \Leftrightarrow u(y)>u(x)$, para todo $x, y \in X$.

Prova: Vide Utility Theory for Decision Making, Peter C. Fishburn, pag. 14.

Definição A.6 Seja $S$ uma relação binária em $X$, então $Y \subseteq X$ é S-ordem denso em $X$ se, e somente se, sempre que $(x, y) \in S$ e $x, y$ são elementos de $X$ mas não de $Y$, existe um $z \in Y$ tal que $(x, z) \in S$ e $(z, y) \in S$.

\section{EXEMPLO 4}

Para a relação de ordem usual da reta real, $R$, o conjunto dos números racioanis, $Q$, é <-ordem denso em $R$.

Teorema A.3 Existe uma função utilidade u : $X \rightarrow R$ tal que $\forall x, y \in X, y \succ x \Leftrightarrow$ $u(y)>u(x)$. Se, e somente se, $\succeq$ é uma ordem fraca em $X$ e existe um subconjunto contável $Y$ de $X / \approx$ onde $Y e ́ \succeq '$-ordem denso em $X / \approx$.

Prova: Vide Utility Theory for Decision Making, Peter C. Fishburn, pag. 27. 


\section{Apêndice B}

\section{S-Convexidade: definições e lemas}

S-bolas, S-convexidade

$S$-bola

Definição B.1 Seja ${ }^{*} \rho$ extensão nã "standard" da métrica usual em $R^{n}$. O conjunto dos pontos $S(x, \delta) \mathrm{em}^{*} R^{n}$ é chamado de $S$-bola, se existe $x \in{ }^{*} R^{n}$ e um real standard positivo, $\delta$, tal que:

$$
S(x, \delta)=\left\{y: \circ\left({ }^{*} \rho(x, y)\right)<\delta\right\}
$$

Chama-se S-Topologia, a topologia gerada pelas S-bolas.

Definição B.2 O "S-convex hull", de um conjunto $A$, é o conjunto de todas as combinaçôes lineares convexas finitas dos elementos de A. Isto é, o conjunto dos elementos $y=\sum_{j=1}^{n} \alpha_{j} x_{j}, x_{j} \in A, \forall j=1, \ldots, n ; e \sum_{j=1}^{n} \alpha_{j}=1$, onde $\alpha_{j} \in{ }^{*} R, \forall j=1, \ldots, n$; $n \in N$ e $\alpha_{i} \geq 0$.

Um conjunto $A$ é dito $S$-convexo se "S-convex hull" de A está contido em $A$.

Definição B.3 Um conjunto $D$ é dito "nearstandard" se para todo $x \in D, x$ é finito.

Definição B.4 OS-interior de um conjunto $A$ de ${ }^{*} R^{n}, S$-int $(A)$ é a união de todas os subconjuntos que são abertos na S-topologia. 
Lema B.1 Se $A$ é um subconjunto de ${ }^{*} R^{n}$, e $0 \notin S$-int $(A)$, então para todo $x \in S$-int $(A)$, $\mu(x) \cap \mu(0)=\emptyset$

Prova: Suponha que exista $x \in S$-int $(A)$ tal que $\mu(x) \cap \mu(0) \neq \emptyset$. Tome $y \in \mu(x) \cap \mu(0)$, temos $y=\varepsilon_{1}$ e $y=x+\varepsilon_{2}, \varepsilon_{1}, \varepsilon_{2} \in \mu(0)$. Vem daí que ${ }^{*} \rho(0, x)={ }^{*} \rho\left(y-\varepsilon_{1}, y-\varepsilon_{2}\right) \approx$ $0 \in \mu(x)$. Como $x \in S$-int $(A)$, exite $\delta>0, \delta \in R$, tal que $\mu(x) \subset S(x, \delta) \subset A$ e portanto $0 \in S$-int $(A)$, contra hipótese.

Lema B.2 Se $A$ é um subconjunto de ${ }^{*} R^{n}, 0 \notin S-\operatorname{int}(A)$, entâo $0 \notin{ }^{\circ}(S-\operatorname{int}(A))=$ $\left\{{ }^{\circ} x: x \in S-\operatorname{int}(A), x\right.$ finito $\}$.

Prova: Se $0 \in{ }^{\circ}(S-\operatorname{int}(A)), \exists x \in S-\operatorname{int}(A)$ tal que $0 \in \mu(x)$ e portanto $\mu(0) \cap \mu(x) \neq \emptyset$, o que contradiz o lema 1.

Os lemas 3 e 4 a seguir tem por objetivo mostrar que o $S$-interior de um $S$-convexo em ${ }^{*} R^{n}$ é $S$-convexo. Para isto, vamos adaptar a prova de que o interior de um conjunto convexo em $R^{n}$ é convexo. Primeiramente, vamos acrescentar mais algumas notações, e conceitos envolvendo as $S$-bolas.

A $S$-bola fechada será denotada por $\bar{S}(x, a)$.

A $S$-bola fechada de centro 0 e raio 1 será denotada simplesmente por $B$. Desta forma $S(x, a)=x+\alpha B$.

O $S$-fecho de conjunto $A$ é o complementar do $S$-interior do complemetar de $A$.

Observação: Dado um conjunto $A$, o $S$-fecho de $A, S$-cl( $A)$, e o $S$-interior de $A, S$-int $(A)$, podem ser expressos como segue:
a) $S-\operatorname{cl}(A)=\cap\{A+\alpha B: \not{\not} 0\}$;
b) $S$-int $(A)=\{x: \exists \alpha \not{\not} 0, x+\alpha B \subset A\}$. 
Prova de b)

Suponha $x \in S$-int $(A)$, temos que existe $\lambda>0, \lambda$ real "standard" tal que a $S(x, \lambda) \subset$ $A$, logo tomando $\delta=\frac{1}{2} \lambda$, temos a $\bar{S}(x, \delta) \subset S(x, \lambda) \subset A$. E sendo $\bar{S}(x, \delta)=x+\delta B$, temos $S$-int $(A) \subseteq\{x>\exists \delta \not{\approx} 0, x+\delta B \subset A\}$.

$$
\{x: \exists \delta \not{\approx} 0, x+\delta B \subset A\} \subseteq S \text {-int }(A)
$$

Prova: imediata.

\section{Prova de a)}

Suponha $x \in S$-cl $(A)$, temos $x$ pertence ao $S$-interior de $A, S$-int $(A)$, ou $x$ pertence a $S$ Fronteira de $A, S-\operatorname{Fr}(A)$. Se $x \in x+\delta B$, para todo $\delta \not \approx 0$, e portanto $x \in \cap\{A+\delta B, \delta \not 0\}$, de forma análoga se $x \in S$ - $\operatorname{Fr}(A)$, temos $x+\lambda B \cap A \neq \emptyset$, para todo $\lambda \not \approx 0$, e portanto existe $y \in A$ e existe $\delta \not{\approx} 0$ tal que $x \in y+\delta B, \log S \operatorname{ccl}(A) \subseteq \cap\{A+\delta B: \not \approx 0\}$.

$\cap\{A+\delta B, \delta \not \approx 0\} \subset S-\mathrm{cl}(A)$, prova é imediata.

Lema B.3 Seja A um conjunto S-Convexo de ${ }^{*} R^{n}$. Seja $x \in S$-int. $(A)$ e y $\in S$-cll $(A)$. Então $(1-\lambda) x+\lambda y$ pertence ao $S$-int. $(A)$ para $0 \leq \lambda \not \varkappa 1$.

Prova: : (obs: basta provar $\lambda$ real "standard", pois para todo $\theta \approx \lambda$, tem-se que

$$
z=(1-\lambda) x+\lambda y \approx(1-\theta) x+\theta y)
$$

Seja $\lambda$ real "standard", $\lambda \in[0,1)$. Afirmamos que $(1-\lambda) x+\lambda y+\delta B \subset A$ para algum "standard" $\delta>0$. Temos que para todo $y \in S-\operatorname{cl}(A) \Rightarrow y \in A+\delta B, \forall \delta>0$. Assim, para todo $\delta>0$,

$(1-\lambda) x+\lambda y+\delta B \subset(1-\lambda) x+\lambda(A+\delta B)+\delta B=(1-\lambda)\left[x+\delta(1+\lambda)(1-\lambda)^{-1} B\right]+\lambda A$, pois para todo $z$ tal que $z \in(\lambda+1) B$, temos $z=(\lambda+1) m, m \in B$, e portanto $z=$ $m \lambda+m \in \lambda+B \Rightarrow(\lambda+1) B \subseteq \lambda B+B$, agora se $z \in \lambda B+B \Rightarrow \exists m, n \in B$, tal que 
$z=\lambda m+n$, onde $\lambda m \in \lambda B$, vem daí que

$$
y=\frac{1}{(\lambda+1)} z=\frac{1}{(\lambda+1)}(\lambda m+n) \in B
$$

pois

$$
\begin{aligned}
& { }^{*} \rho(0, y)={ }^{*} \rho\left(0, \frac{\lambda}{(\lambda+1)} m+\frac{1}{(\lambda+1)} n\right) \leq \frac{\lambda}{(\lambda+1)}^{*} \rho(0, m)+\frac{1}{(\lambda+1)}{ }^{*} \rho(0, n) \\
& \Varangle \frac{\lambda}{(\lambda+1)}+\frac{1}{(\lambda+1)}=1 \Rightarrow(\lambda+1) y=z \in(\lambda+1) B \\
& \Rightarrow(\lambda+1) B+B \subseteq(\lambda+1) B \text {, }
\end{aligned}
$$

logo, $(\lambda+1) B+B=(\lambda+1) B$, o que prova a igualdade acima. Mas $x+\delta(1+\lambda)(1-\lambda)^{-1} B \subset$ $A$ para $\delta$ suficientemente pequeno. Entretanto, $(1-\lambda) x+\lambda y+\lambda B \subset(1-\lambda) A+\lambda A=A$ para algum $\delta>0$.

Lema B.4 Se A é um $S$-convexo "nearstandard" de ${ }^{*} R^{n}$, então, $S$-int $(A)$ é $S$-convexo.

Prova: : Tome $y \in S$-int $(A)$, pelo lema 3 , temos para $0 \leq \lambda \not \approx$, para todo $x \in S$-int $(A)$, temos: $(1-\lambda) x+\lambda y \in S$-int $(A)$. Mas para $\lambda \approx 1$, temos $(1-\lambda) x+\lambda y \approx y$ e como $y \in S$-int $(A)$, segue que $S$-int $(A) S$-convexo.

Lema B.5 Se $A$ é um conjunto $S$-convexo de ${ }^{*} R^{n}$, então ${ }^{\circ} A$ é um conjunto convexo de $R^{n}$

Prova: Sejam $x, y \in{ }^{\circ} A$ e $\alpha$ número real "standard", tal que $0 \leq \alpha \leq 1$. Então existem infinitesimais $\varepsilon_{1}, \varepsilon_{2}$, tais que $x+\varepsilon_{1}, y+\varepsilon_{2} \in A$. Mas $A$ é $S$-convexo e portanto $A \ni$ $\alpha\left(x+\varepsilon_{1}\right)+(1-\alpha)\left(y+\varepsilon_{2}\right)=\alpha x+(1-\alpha) y+\alpha \varepsilon_{1}+(1-\alpha) \varepsilon_{2}$ e sendo $0 \approx \alpha \varepsilon_{1}+(1-\alpha) \varepsilon_{2}$. Segue que $\alpha x+(1-\alpha) y \in{ }^{\circ} A$, o que prova a afirmação.

Lema B.6 Lema da $S$-separação Se A é um conjunto "nearstandard" $S$-convexo de ${ }^{*} R^{n}$ $e 0 \notin S$-int $(A)$, então existe um vetor "standard" $p \neq 0$ tal que para todo $y \in S$-int $(A)$, p.y $\not 20$. 
Prova: Pelo lema 4, $S$-int $(A)$ é convexo. Pelo lema $5,^{\circ}(S-\operatorname{int}(A))$ é um convexo de ${ }^{*} R^{n}$. Pelo lema $2,0 \notin^{\circ}(S$-int $(A))$. Portanto, existe $p \in R^{n}$ tal que $p \neq 0$ e para todo $x \in{ }^{*}(S$ $\operatorname{int}(A)), p . x>0$ (vide p.59, teorema 3.4, Functional analysis; Rudin, Walter. Second Edition; McGraw-Hill, inc.), todo $y \in S$-int( $(A)$ pode ser expresso como $y=x+\varepsilon$ onde $x \in{ }^{\circ}(S$-int $(A))$ e $\varepsilon \approx 0$. Assin, para todo $y \in S$-int $(A)$, temos que $p \cdot y+p \cdot \varepsilon \Rightarrow p \cdot y \not 0$. 


\section{Apêndice $\mathrm{C}$}

\section{Elementos de análise não "standard"}

Apresentamos aqui uma introduçã às técnicas da Análise não "standard". Para evitar entrar em técnicas de lógica foi utilizado um enfoque mais categórico, e são omitidas as provas de que tais axiomatização é consistente, omitiremos também as provas dos t.eoremas, tais provas podem ser encotradas em trabalho publicado no 42 o Seminário Brasileiro de Análise, p.77 a 147. Com isto é possível trazer este assunto a um público não familiarizado com o cálculo de predicados, mas apenas maturidade matemática de um aluno de mestrado em matemática.

As ferramentas da Análise não "standard"

A expressão "Análise não Standard" designa a formalização que Abraham Robinson fez na década de 1960 das idéias de Leibnitz acerca de fazer o cálculo com os "infinitésimos". Leibnitz introduziu esses elementos infinitésimos (e também seus inversos, os elementos infinitamente grandes) como elementos ideais que deviam possuir as "mesmas propriedades" de R. Mas surgia um problema na hora de eliminá-los, desprezando os ditos infinitésimos de "ordem superior". Por exemplo, quando se escrevia $d f(x)=f^{\prime}(x) d x$, querendo dizer que um incremento infinitesimal em $x$ resultava em um incremento linear em $f(x)$ da forma $f^{\prime}(x) d x$, desprezando-se os incrementos de ordem superior (ou seja, em $(d x)^{2},(d x)^{3}$, etc.). 
Então, a igualdade ficava descaracterizada, ficando apenas uma igualdade aproximada. A solução encontrada por A. Robinson foi trabalhar com uma extensão. ${ }^{*} R$, de $R$, contendo tais elementos ideais, e substituindo a igualdade por uma relação de equivalência (a relação "infinitamente próximo").

Apresentamos a seguir o contexto em que é feita a análise não-standard".

\section{Superestruturas}

O universo em que faremos a análise não "standard" consiste em duas coleções (ou categorias) de conjuntos, chamadas de superestrutura, e que são relacionadas por uma aplicação (ou funtor) satisfazendo algumas propriedades. Tais superestruturas serão denotadas por $V_{\text {st }}$ (o mundo "standard"), contendo $R$, os reais standard e $V_{n_{s}}$ (o mundo não standard), contendo um conjunto * $R$, conjunto dos reais não "standard". Denotando ambas indistintamente por $V$, elas devem satisfazer as seguintes propriedades:

1) Se $A, B \in V$, então $A \times B \in V$;

2) Se $A \in V$ e $B \subset A$, então $B \in V$, e também o conjunto das partes de $A, P(A) \in V$;

3) Se $A, B \in V$, então o conjunto das funções de $A \operatorname{em~} B, \operatorname{Fun}(A, B)$, está em $V$.

A conexão entre essas categorias é dada por uma aplicação $*: V_{s l} \rightarrow V_{n s}$ (chamada de monomorfismo) que leva conjuntos $A$ a conjuntos ${ }^{*} A$, funções $f: A \rightarrow B$ a funções ${ }^{*} f: A \rightarrow{ }^{*} B$ e satisfaz:

1) ${ }^{*}(f(a))={ }^{*} f\left({ }^{*} a\right)$, para todo $a \in A$;

2) ${ }^{* i} d_{A}=i d_{* A}$ (i.e, * leva a função identidade de $A$ à função identidade de ${ }^{*} A$ ), e * comuta com a composição, ou seja, ${ }^{*}(g \circ f)={ }^{*} g \circ{ }^{*} f$ (em outras palavras, $*$ é um funtor); 
3) ${ }^{*}\left(A_{1} \times A_{2}\right)={ }^{*} A_{1} \times{ }^{*} A_{2}$, e se $p_{i}: A_{1} \times A_{2} \rightarrow A_{i}$ for a projeção sobre a $i$-ésima coordenada, então ${ }^{*} p_{i}:{ }^{*} A_{1} \times{ }^{*} A_{2} \rightarrow{ }^{*} A_{i}$ será também a projeção sobre a $i$-ésima coordenada;

4) Sejam $A_{1}, \ldots, A_{m}, B \in V, m \in N$, denotemos $A=A_{1} \times \cdots \times A_{m}$; dadas $f_{i}, g_{i}: A \rightarrow$. $B, i=1, \ldots, n$, sejam $S(x)$ e ${ }^{*} S(x)$ os seguintes sistemas de equaçōes, desigualdades e relações de pertinência, $\operatorname{com} x=\left(x_{1}, \ldots, x_{m}\right)$ :

$$
S(x):\left\{\begin{array}{ll}
f_{i}(x)=g_{i}(x) & , 1 \leq i \leq j \\
f_{i}(x) \neq g_{i}(x) & , j<i \leq 1 \\
f_{i}(x) \in g_{i}(x) & , 1<i \leq p \\
f_{i}(x) \notin g_{i}(x) & , p<i \leq n
\end{array} \quad{ }^{*} S(x): \begin{cases}{ }^{*} f_{i}(x)={ }^{*} g_{i}(x) & , 1 \leq i \leq j \\
{ }^{*} f_{i}(x) \neq{ }^{*} g_{i}(x) & , j<i \leq 1 \\
{ }^{*} f_{i}(x) \in{ }^{*} g_{i}(x) & , 1<i \leq p \\
{ }^{*} f_{i}(x) \notin{ }^{*} g_{i}(x) & , p<i \leq n\end{cases}\right.
$$

Então, se ${ }^{*} S(x)$ tiver solução em ${ }^{*} A$ então $S(x)$ terá solução em $A$;

5) Suporemos também que ${ }^{*} R$ seja uma extensão própria de $R$, ou seja, o conjunto dos elementos ${ }^{*} r(r \in R)$ será um subconjunto próprio de ${ }^{*} R$.

Vamos mostrar que essa aplicaçao preserva as propriedades nas superestruturas, no seguinte sentido: toda proposição de $V_{s t}$ é verdadeira (no mundo "standard") se, e somente se, a proposição obtida "estrelando-se" esses elementos for verdadeira no mundo não "standard". Em particular, ${ }^{*} R$ terá as mesmas propriedades de $R$ : entre outras coisas, será um corpo ordenado. Tais proposiçes são afirmaçes sobre combinações booleanas dos conjuntos das soluçes de sistemas $S_{1}(x), \ldots, S_{k}(x), x=\left(x_{1}, \ldots, x_{n}\right)$, obtidas quantificandose as variáveis $x_{1}, \ldots, x_{n}$. Não perdemos em generalidades supondo que as funçes que compem esses sistemas tm o mesmo domínio e mesmo contradomínio, pois poderiamos introduzir variáveis redundantes para transformarmos sistemas com domínios distintos no caso acima. Para formarmos as proposiçes, restringir-nos-emos às quantificações das formas "exite $x_{i}$ " e "para todo $x_{i}$ ", e denotaremos uma quantificação em $x_{i}$ por $Q_{i} x_{i}$.

Teorema C.1 (Princípio de transferência) Com as conveçõe acima valem as seguintes afirmações: 
1) se $B=\left\{a \in A_{m-k+1} \times \cdots \times A_{m}: Q_{1} x_{1}, \cdots Q_{m-k} x_{m-k}\right.$ (uma combinação booleana $\left.\left.\operatorname{dos} S_{j}(\vec{x}, a)\right)\right\}$, então ${ }^{*} B=\left\{a \in{ }^{*} A_{m-k+1} \times \cdots \times{ }^{*} A_{m}: Q_{1} x_{1} \cdots Q_{m-k} x_{m-k}\right.$ (a mesma combinaçâo booleana nos correspondentes $\left.\left.{ }^{*} S_{j}(\vec{x}, a)\right)\right\}$;

2) uma proposição da forma " $Q_{1} x_{1} \cdots Q_{m} x_{m}$ (uma combinação booleana dos $S_{j}(\vec{x}, a)$ )" é verdadeira "no mundo "standard" se, e somente se, a versào "estrelada", " $Q_{1} x_{1}$, $\ldots, Q_{m} x_{m}$ (a mesma combinaçâo booleana dos correspondentes ${ }^{*} S_{j}(\vec{x} . a)$ )" for verdadeira no mundo não "standard".

Lema C.1 Propriedades descritas por frases da forma "existe y tal que $Q(x, y)$ ", em que $Q(x, y)$ é uma combinação booleana de sistemas de equaçes e desigualdades. como acima, também podem ser descritas por uma combinação booleana de sistema $S^{\prime}(x)$.

Os reais nào "stantard"

Infinitésimos e elementos ilimitados

Como a extensão é própria, ${ }^{*} R$ não é arquimediano, ou seja, existem elementos $\delta \in{ }^{*} R$ tais que $0<|\delta|<1 / n$, para todo $n \in N, n>0$. Tais elementos (junto com o zero) são chamados de infinitésimos. Se $m(0)$ denota o conjunto dos infinitésimos, podemos definir uma relação $\approx \mathrm{em}^{*} R$, dada por $x \approx y$, se $x-y \in m(0)$. Esta é claramente uma relação de equivalência, que respeita as operações de soma e produto. É com esta relação que faremos os seguintes argumentos não "standard". Observe que o inverso de um infinitésimo positivo não nulo é maior que qualquer elemento de $R$.

Mônadas e galáxias

Sejam. $G(0)=\{x \in R: \exists y \in R /|x|<y\}$, o conjunto dos elementos limitados; $m(0)=\left\{x \in{ }^{*} R: \forall n \in N(|x|<1 / n)\right\}$, o conjunto dos infinitésimos (em relação a $R$ ); e os elementos de ${ }^{*} R_{\infty}={ }^{*} R \backslash G(0)$ serão ditos ilimitados. Dado $a \in R$ definimos a mônada de a por $m(a)=\left\{x \in{ }^{*} R: \forall n \in N(|x-a|<1 / n\}\right.$, e a galáxia de $a$ por 
$G(a)=\left\{x \in{ }^{*} R: \exists y \in R|| x-a \mid<y\right\}$. É claro que $m(a)=m(0)+a$ e $G(a)=G(0)+a$. A relação "infinitamente próximos"

Se $x, y \in{ }^{*} R$, dizemos que $x$ e $y$ estão infinitamente próximos, ou $x \approx y$, se $x-y \in$ $m(0)$.

Teorema C.2 Se $x \in G(0)$, então existe um único $y \in R$ tal que $x \approx y$.

A parte "standard"

Este teorema permite definir a função ${ }^{\circ}: G(0) \rightarrow R$, sendo ${ }^{\circ} x$ o único $y \in R$ tal que $x \approx y$, esta é dita aplicação parte "stantard".

Os naturais não "standard"

Lembremos que ${ }^{*} N$ representa o conjunto $\left\{x \in{ }^{*} R:{ }^{*} \chi_{n}(x)=1\right\}$. Vamos mostrar que ${ }^{*} N$ é uma extensão própria de $N$. Este será um primeiro exercício na aplicação do princípio de transferência.

Lema C.2 Existe $x \in{ }^{*} N$, tal que $x \notin N$.

Prova: Sabemos que para todo $x \in R$, existe $y \in N$ tal que $x<y$. Aplicando-se o princípio de transferência para a sentença, e usando a representação da relação de pertinência, descrita acima, obtemos que será verdade $\mathrm{em}{ }^{*} R$ a seguinte afirmação: para todo $x \in{ }^{*} R$, existe $y \in{ }^{*} N$, tal que $x<y$. Como ${ }^{*} R$ possui elementos infinitamente grandes, ${ }^{*} N$ também possuirá elementos infinitamente grandes.

\section{Conjuntos internos e externos}

Definição C.1 Dizemos que um elemento $A$ é interno se existe $B \in V(X)$ tal que $A \in B$. Caso contrário, diremos que $A$ é externo. Em particular, todo conjunto da forma ${ }^{*} B$ é interno, pois vale $B \in \mathcal{P}(B)$, então vale ${ }^{*} B \in{ }^{*} \mathcal{P}(B)$ (nas respectivas superestruturas). Diremos que uma função é interna se o seu gráfico for um conjunto interno. 
Teorema C.3 (Keisler) Se $S_{1}(\vec{x}), \ldots, S_{k}(\vec{x})$ são sistemas cujas funções são internas (e portanto, seus domínios e contra domínios sâo conjuntos internos), então $X=\{a \in$ $A_{m-k+1} \times \cdots \times A_{m}: Q_{1} x_{1} \cdots Q_{m-k} x_{m-k}$ (uma combinação booleana dos $\left.\left.S_{j}(\vec{x})\right)\right\}$ é um conjunto interno.

Teorema C.4 (Princípio de permanência) Seja $\phi\left(y, x_{1}, \ldots, x_{n}\right)$ uma fórmula descrevendo uma propriedade (a partir de sistemas e quantificaçôes), e sejam $a_{1}, \ldots, a_{x}$ elementos internos. Então:

1) se cada $x \in N$ (ou $x \in{ }^{*} R \backslash^{*} R_{\infty}$, e $x>0$ ) satisfaz $\phi\left(x, a_{1}, \ldots, a_{n}\right)$, então existe $k \in{ }^{*} N_{\infty}\left(={ }^{*} N \cap{ }^{*} R_{\infty}\right)$ (ou respectivamente, $k \in{ }^{*} R_{\infty}$ e $k>0$ ), tal que todo $b \leq k$ em ${ }^{*} N$ (respectivamente em ${ }^{*} R$ ) satisfaz $\phi(b, \vec{a})$

2) se cada $x \in{ }^{*} N_{\infty}\left(\right.$ ou $\left.x \in{ }^{*} R_{\infty}, e x>0\right)$ satisfaz $\phi\left(x, a_{1}, \ldots, a_{n}\right)$, entũo existe $k \in N$ (ou, respectivamente, $k \in R$ e $k:>0$ ), tal que todo $b \geq k$ em ${ }^{*} N$ (respectivamente, ${ }^{*} R$ ) satisfaz $\phi(b, \vec{a})$.

3) se cada $x \in m(0), x>0$, satisfaz $\phi\left(x, a_{1}, \ldots, a_{n}\right)$, entâo existe $k \in R, k>0$, tal que para todo $b \in{ }^{*} R, 0<b<k$, b satisfaz $\phi(b, \vec{a})$.

Teorema C.5 (Princípio de esparramamento) Seja A um subconjunto interno de ${ }^{*} R$. Então:

1) se A contém todos os naturais "standard" então A contém um natural não "standard".

2) se A contém todos os naturais não "standard" entâo A contém algum natural "standard".

3) se A contém todos os infinitésimos positivos, então A contém um número real "standard" positivo. 
Definição C.2 Seja $\mathcal{P}_{F}(A)$ conjunto dos subconjuntos finitos de $A \in V(X)$. Dizemos que $B \in V\left({ }^{*} X\right)$ é hiperfinito se existe $A \in V(X)$, tal que $B \in{ }^{*} \mathcal{P}_{F}(A)$.

Teorema C.6 Seja $B \in V\left({ }^{*} X\right)$ hiperfinito. Entâo existem $j \in{ }^{*} N$ e bijeção interna $f: J \rightarrow B$, onde $J$ é um intervalo interno $\left\{k: \in{ }^{*} N: k \leq j\right\}$.

Definição C.3 $V\left({ }^{*} X\right)$ é um alargamento de $V(X)$ se para todo $A \in V(X)$ existe $B \in$ ${ }^{*} \mathcal{P}_{F}(A)$ tal que para todo $a \in A\left({ }^{*} a \in B\right)$.

Definição C.4 Uma relação binária $P$ é dita concorrente (ou entâo finitamente satisfativel) em $A \subseteq \operatorname{dom}(P)$ se para todo subconjunto finito $\left\{z_{1}, \ldots, z_{n}\right\}$ de $A$, existe $y \in$ $\operatorname{codom}(P)$ tal que $\left(x_{i}, y\right) \in P, 1<i \leq n$. Dizemos que $P$ é concorrente se o for em $\operatorname{dom}(P)$.

Teorema C.7 São equivalentes:

1) $V^{*}(X)$ é um alargamento;

2) para toda relação concorrente $P \in V(X)$, existe $y \in \operatorname{codom}(P)$, tal que $\left({ }^{*} x, y\right) \in{ }^{*} P$, para todo $x \in \operatorname{dom}(P)$.

Saturação por abrangência

Definição C.5 Seja $\kappa$ um cardinal infinito. Dizemos que o morfismo ${ }^{*}: V(X) \rightarrow V(Y)$ é $\kappa$-abrangente se para todos $C, D \in V(X)$ e função $h: C \rightarrow D$, existe $g:{ }^{*} C \rightarrow{ }^{*} D$ interna, tal que, para todo $a \in C, h(a)=g(a)$.

Definição C.6 Dizemos que $V(Y)$ é $\kappa$-saturada se para toda famitia de conjuntos internos de $V(Y),\left\langle A_{i}\right\rangle_{t \in J}$, com $|J|<\kappa$, cujas subfamílias finitas têm cada uma interseção não vazia (ou seja, tem a pif-propriedade da interseçào finita), temos que $\cap\left\langle A_{i}\right\rangle_{t \in J} \neq \emptyset$. 
Teorema C.8 Dado um cardinal regular infinito $\kappa$, existe ${ }^{*} X$ tal que $V\left({ }^{*} X\right)$ é $\kappa$-saturado.

Relação entre saturação, abrangência e alargamento

Teorema C.9 Considere a aplicaçâo ${ }^{*}: V(X) \rightarrow V\left({ }^{*} X\right), \operatorname{com} V\left({ }^{*} X\right)$ ґ-saturado. Sejam $C, D \in V\left({ }^{*} X\right)$, com $D$ interno, os elementos de $C$ são internos (embora $C$ não seja necessariamente interno), e $|C|<\kappa$. Seja $\phi: C \rightarrow D$ uma função qualquer. Então, existe $\tilde{C}$ interno, com $\tilde{C} \subseteq C$, e uma função interna $\tilde{\phi}: \tilde{C} \rightarrow D$ estendendo $\phi$. Se $C=\left\{{ }^{*} a: a \in C_{0}\right\}, C_{0} \in V(x)$, então podemos tomar $\tilde{C}={ }^{*} C_{0}$.

Teorema C.10 Seja $\kappa>|V(X)|$, e suponha que $V\left({ }^{*} X\right)$ seja $\kappa$-saturada. Então, $V\left({ }^{*} X\right)$ também é um alargamento. 


\section{Bibliografia}

Araújo, P.A. [1983]. Introdução à economia matemática. 14º Colóquio Brasileiro de Matemática, IMPA, RJ.

Arrow, J.K.; Intriligator, H.D. [1991]. Handbook of mathematical economics, v.1 e 2, North Holland, Amsterdam.

Brown, J.D. and Robinson, A.[1975]. Nonstandard exchange economics. Econometrica, 41-55.

Brown, J.D. and Robinson, A. [1974]. The cores of large standad exchange economies. Journal of Economic 9, 245-254.

Debreu, G. [1971]. Theory of value, an axiomatic analysis of economic equilibrium. Cowles Foundation, New Haven.

Eatwel, J.; Milgate, M.; Newman, P. [1989]. General equilibrium. The New Palgrave, W.W. Norton, London.

Fishburn, P.C. [1970]. Utility theory for decision making. John Wiley \& Sons, Inc., New York.

Hildebrand, W. [1974]. Core and equilibria of a large economy. Princeton University Press, New Jersey.

Hildebrand, W.; Kirman, P.P. [1991]. Equilibrium analysis. North Holland, Amsterdam.

Lages, E. [1984]. Curso de análise, v.2. Instituto de Matemática Pura e Aplicada, IMPA-CNPq, RJ

Mas Colell, A. [1976]. The theory of general economics equilibrium, a differentiable approach. Cambridge University Press. 
Prandini, J.C.; Bianconi, R.; Raymundo, C.A.B. [1995]. Introdução à análise não "standard”. 42ํㅡolóquio Brasileiro de Análise, CNPq, FAPESP, SBM, Maringá-PR.

Robinson, A. [1974]. Nonstandard analysis. Revised edition. North Holland Publishing Company, Amsterdam.

Rudin, W. [1991]. Functional analysis. 2.ed. McGraw Hill, Inc., Singapore.

Stroyan, K.D.; Luxemburg, W.A.J. [1976]. Introduction to the theory of infinitesimals. Academic Press, New York. 